\title{
Promising applications of aggregation- induced emission luminogens in organic optoelectronic devices
}

\author{
Maoxing Yu ${ }^{1 \dagger}$, Ruishan Huang ${ }^{1 \dagger}$, Jingjing Guo ${ }^{1}$ Zujin Zhao ${ }^{1 *}$ and Ben Zhong Tang ${ }^{1,2^{*}}$
}

\author{
*Correspondence: mszjzhao@scut. \\ edu.cn; tangbenz@ust.hk \\ ${ }^{\dagger}$ Maoxing Yu and Ruishan Huang \\ contributed equally to this work. \\ 'State Key Laboratory of \\ Luminescent Materials and Devices, \\ Guangdong Provincial Key \\ Laboratory of Luminescence from \\ Molecular Aggregates, South China \\ University of Technology, \\ Guangzhou 510640, China \\ Full list of author information is \\ available at the end of the article
}

\begin{abstract}
Since the first report of aggregation-induced emission (AIE) concept in 2001, it has received intense attentions from academy and industry because of its important applications in diverse research fronts. Up to now, the luminogens with AlE property (AlEgens) have been widely used in optoelectronic devices, fluorescent bioprobes and chemosensors, and researchers have also committed to exploring the potentials of AlEgens in other cross-cutting areas. The AlEgens have shown superior advantages such as highly efficient emissions in the aggregated state and thus exhibited better performances in comparison with traditional luminescent materials whose emissions are usually quenched upon aggregate formation. In view of the significant achievements of AlEgens in recent years, this review presents representative advancements of AlEgens for the applications in organic optoelectronic devices, mainly including organic light-emitting diodes (OLEDs), circularly polarized luminescence (CPL) devices, electrofluorochromic (EFC) devices, luminescent solar concentrators (LSCs), and liquid crystal displays (LCDs). Not only the design strategies of AlEgens for these optoelectronic devices are analyzed, but also their structureproperty relationship and working mechanism are elucidated. It is foreseeable that robust AlEgens with specific functionalities will find more and more applications in various research fields and play an increasingly important role in high-tech devices.

Keywords: Aggregation-induced emission, Thermally activated delayed fluorescence, Aggregation-induced delayed fluorescence, Organic light-emitting diode, Circularly polarized luminescence, Electrofluorochromism, Luminescent solar concentrator, Liquid crystal display
\end{abstract}

\section{Introduction}

The progress of the contemporary society is inseparable from exploitation of semiconductor industry [1]. Since the twentieth century, from the vacuum tube to the very large-scale integration circuit, the inorganic semiconductors have been developed rapidly and applied in all aspects of human life. However, conventional optoelectronic components using inorganic semiconductors are built on the rigid substrates and require complex processing technique with high energy consumption, which gradually become unfavored in the present energy-constrained world. In contrast, organic

(c) The Author(s). 2020 Open Access This article is licensed under a Creative Commons Attribution 4.0 International License, which permits use, sharing, adaptation, distribution and reproduction in any medium or format, as long as you give appropriate credit to the original author(s) and the source, provide a link to the Creative Commons licence, and indicate if changes were made. The images or other third party material in this article are included in the article's Creative Commons licence, unless indicated otherwise in a credit line to the material. If material is not included in the article's Creative Commons licence and your intended use is not permitted by statutory regulation or exceeds the permitted use, you will need to obtain permission directly from the copyright holder. To view a copy of this licence, visit http://creativecommons.org/licenses/by/4.0/. 
optoelectronic devices possess simple processing technique, flexibility, light-weight, environment-friendly and cost-effective advantages that have broader development prospects in some specific areas. And they have achieved remarkable progress since the discovery of conducting polymers by Heeger, MacDiarmid and Shirakawa in 1977 [2] and the pioneering work of organic light-emitting diodes (OLEDs) by Tang and Van Slyke in 1987 [3]. To date, the optoelectronic devices based on organic materials have been successfully applied in the fields of electronics [4], nonlinear optics [5], displays [3], solar cells [6], sensors [7], storage [8], and so forth.

In order to realize a further breakthrough, developing efficient organic luminogens plays a key role for the fabrication of the high-performance optoelectronic devices such as OLEDs, electrofluorochromic (EFC) devices, luminescent solar concentrators (LSCs) and liquid crystal display (LCDs). Many conventional luminescent materials emit brightly in solutions but experience serious quenching problem in the aggregated state or solid phase, which is referred to aggregation-caused quenching (ACQ). The ubiquitous ACQ effect has impeded the progress of many optoelectronic devices, in which organic luminogens are usually used as solid thin films. To mitigate the ACQ problem, various chemical, physical and/or engineering methods have been proposed but often end with little effect. In 2001, an interesting photophysical concept of aggregation-induced emission (AIE) was reported [9], which has changed the ways of thinking for luminescent materials researches. The emissions of AIE luminogens (AIEgens) can be brightened upon aggregate formation, which is essentially opposite to the ACQ effect. Based on the experimental investigation and theoretical calculation, the restriction of intramolecular motion (RIM), including rotation, vibration, twisting, etc., has been rationalized as the main mechanism of the AIE phenomenon [10]. In solution, the active intramolecular motions of AIEgens provide a nonradiative decay channel to dissipate excited state energy, resulting in very low or null emission. When these motions are restricted by weak intermolecular interactions or physical constraint in the aggregated state, the nonradiative decay channels are blocked and the radiative relaxation is promoted. Therefore, the excited state energy can be released as photons, rendering greatly enhanced emission. In some cases, J-aggregate formation, twisted intramolecular charge transfer (TICT), excited state intramolecular proton transfer (ESIPT), etc. also contribute to the AIE effect [11]. Attracted by the unique advantages of highly efficient solid-state emissions of AIEgens, a great many of functional materials with AIE characteristics have been developed $[12,13]$ and successfully used in organic optoelectronic devices [14], biological sensors [15], chemical probes [16], and molecular electronics [17]. In this review, we summarize representative advancements of AIEgens for the applications in OLEDs, EFC, LSC and LCD. The design strategies and working mechanisms of AIEgens for the organic optoelectronic devices are analyzed in detail. From the future perspective, AIEgens will broaden the range of application fields and play an increasingly important role in high-tech devices.

\section{AlEgens for organic light-emitting diodes}

OLEDs have become one kind of the most competitive candidates for future highresolution flexible display and illumination technologies, owing to the distinct merits of 
high contrast ratio, fast response, wide viewing angle, flexibility, light-weight, and so on. OLEDs are thin-film devices consisting of the hole-transporting layer (HTL), electron-transporting layer (ETL), emissive layer (EML), etc., sandwiched between two electrodes. The conversion of electrical current into light takes place in OLEDs by radiative recombination of excitons. A key parameter of OLED performance, external quantum efficiency (EQE), is the ratio of the number of emitted photons outside the device to the number of injected charges. The theoretical EQE can be estimated as a product of four factors, as illustrated in Eq. (1):

$$
\eta_{\mathrm{EQE}}=\gamma \times \varphi_{\mathrm{F}} \times \eta_{\mathrm{r}} \times \eta_{\text {out }}
$$

where $\gamma$ is the charge carrier balance factor, $\phi_{\mathrm{F}}$ is the photoluminescence quantum yield (PLQY), $\eta_{\mathrm{r}}$ is the exciton utilization ratio for radiation, $\eta_{\text {out }}$ is the light outcoupling factor. The $\gamma$ is usually recommended as high as $100 \%$ with suitable device structures. The $\eta_{\text {out }}$ is defined as $20-30 \%$ due to refractive index of different layers, which can be further improved by tuning molecular orientation or adopting out-coupling enhancement structures [18-20], such as microlens arrays, prisms and micro-structured substrates. Different from photoexcitation, singlet and triplet excitons are generated by electron/ hole recombination with 1:3 ratio on the basis of spin statistics under electrical excitation in OLEDs. Therefore, traditional fluorescent OLEDs are limited to 25\% internal quantum efficiency with the theoretically highest EQE of 5-7.5\% [21]. To further improve the efficiency of OLEDs, numerous light-emitting materials with different photophysical processes, such as phosphorescence, hybridized local and charge transfer (HLCT) [22], triplet-triplet fusion (TTF) [23] and thermally activated delayed fluorescence (TADF) [24] have been explored and utilized to harvest the $75 \%$ triplet excitons for luminescence. By introducing AIE property into these different types of emitters, high PLQYs in solid films can be guaranteed to construct stable and efficient OLEDs. Up to now, the emission colors of the OLEDs based on multifunctional AIEgens have covered the entire visible region, greatly contributing to the vitalization of the OLED market.

\section{AlEgens for blue and white OLEDs}

The devices that emit red, green and blue (RGB) primary colors have always been the focus in OLED display [25-27]. With color conversion technology, blue light can also be converted to green and red light. In addition, efficient blue emitters in white light illumination can not only reduce power consumption, but also facilitate the acquisition of higher color temperature and color rendering index (CRI). Nevertheless, due to the high triplet energy and long exciton lifetime, blue emitters still remain as a big challenge in comparison with green and red emitters, and thus blue OLEDs with high efficiency and long operating time are always not satisfactory [28]. To address this issue, the further exploration of robust blue emitters is of high importance. Recently, AIEgens have realized encouraging achievements in constructing blue emitters, and this review will focus on the represitative advancements of blue emitters based on AIEgens, and their performance in blue and white OLEDs.

Anthracene is a traditional building block to construct blue luminescent materials [29]. However, the rigid and planar molecule structure of anthracene is prone to form compact $\pi-\pi$ stacking in solid state, which is usually undesired for light emitting. 
Grafting $t$-butyl groups and AIE-active tetraphenylethene (TPE) onto anthracene can efficiently inhibit tight molecular packing to mitigate emission quenching effect. Then, the corresponding molecule TPE-TAPBI [30] with a twisted conformation was obtained and showed a low PLQY of $3.9 \%$ in tetrahydrofuran (THF) solution. But in film, TPETAPBI radiated blue light at $459 \mathrm{~nm}$ with a high PLQY of $64.2 \%$, exhibiting typical AIE property. Additionally, the introduction of 1,2-diphenylbenzo [d] imidazole (PBI), an important moiety of commercial electron-transporting material 1,3,5-tri(1-phenyl- $1 \mathrm{H}$ benzo[d]imidazol-2-yl)phenyl (TPBi), could balance carrier transport capacity and increase exciton recombination of TPE-TAPBI. As a result, a good electron mobility value of $3.85 \times 10^{-5} \mathrm{~cm}^{2} \mathrm{~V}^{-1} \mathrm{~s}^{-1}$ for TPE-TAPBI was achieved at an electric field of $5.5 \times 10^{5} \mathrm{~V} \mathrm{~cm}^{-1}$. Strong solid-state photoluminescence (PL) intensity and high electron mobility guaranteed its potential device performance. Indeed, the nondoped OLED with a configuration of indium tin oxide (ITO)/1,4,5,8,9,11-hexaazatriphenylenehexacarbonitrile (HATCN) $(5 \mathrm{~nm}) / N, N^{\prime}$-bis(naphthalen-1-yl)- $N, N^{\prime}$-bis(phenyl)-benzidine (NPB) $(40 \mathrm{~nm}) /$ tris $(4$-carbazoyl-9-ylphenyl)amine (TCTA) $(5 \mathrm{~nm}) /$ TPE-TAPBI $(20 \mathrm{~nm}) / 1,3,5$ tri(mpyrid-3-ylphenyl)benzene (TmPyPB) $(40 \mathrm{~nm}) /$ lithium fluoride (LiF) $(1 \mathrm{~nm}) / \mathrm{Al}$

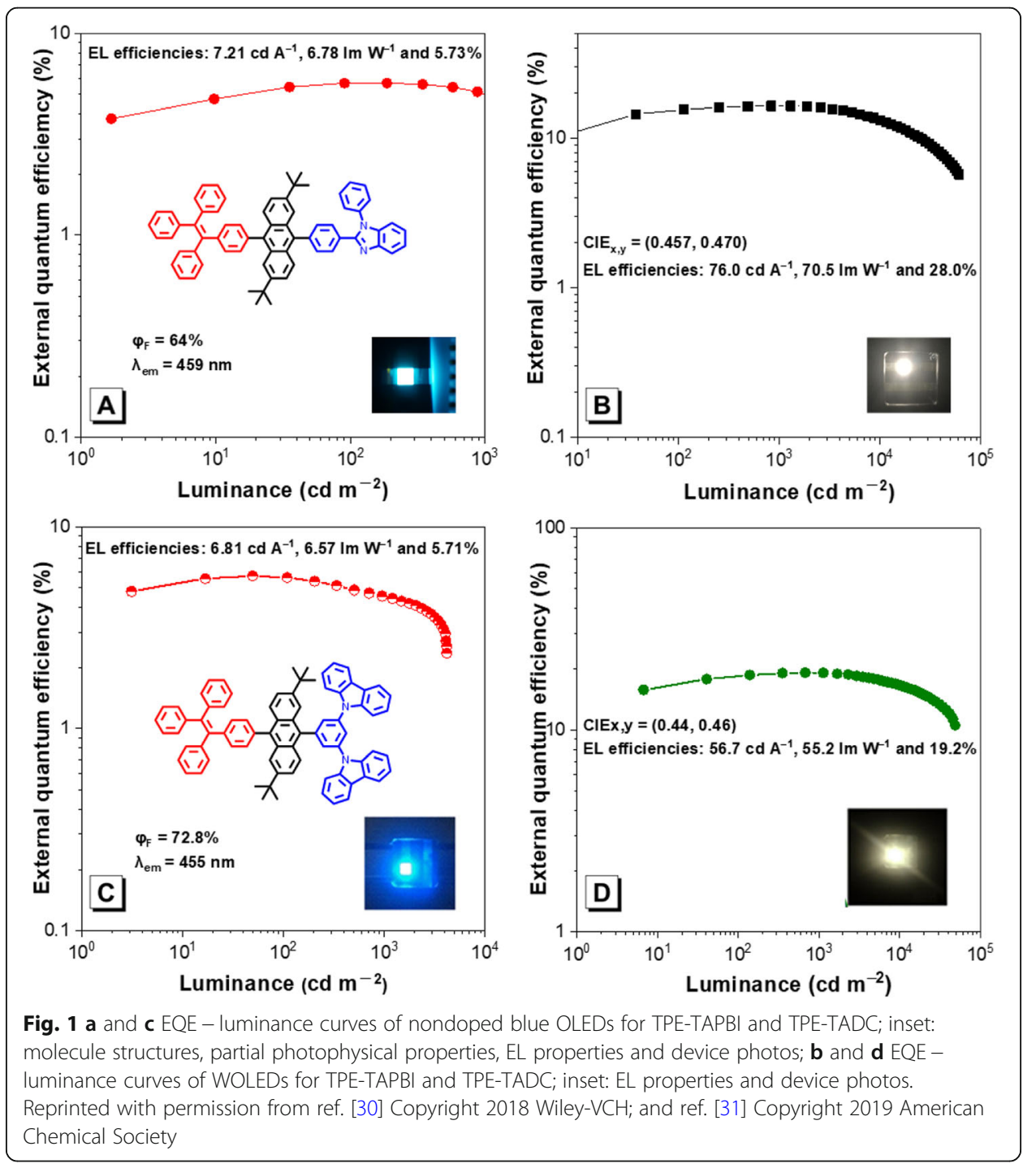


emitted blue light $\left(\mathrm{CIE}_{\mathrm{x}, \mathrm{y}}=0.15,0.16\right)$ with an excellent maximum EQE of $5.73 \%$ (Fig. 1a). It is worth noting that the electroluminescence (EL) efficiencies dropped slowly with a low roll-off at $1000 \mathrm{~cd} \mathrm{~m}^{-2}$. Inspired by the outstanding performance of the nondoped blue OLED, two-color hybrid white OLED (WOLED) was fabricated with a structure of ITO/HATCN $(150 \mathrm{~nm}) / \mathrm{NPB}(15 \mathrm{~nm}) /$ TAPC $(5 \mathrm{~nm}) / \mathrm{NPB}$ : bis[2-(2-hydroxyphenyl)-pyridine]beryllium (Bepp2): bis(2-phenyl-4,5-dimethylpyridinato)[2-(biphenyl-3-yl)pyridinato]iridium (III) $\left[\operatorname{Ir}(\mathrm{dmppy})_{2}(\mathrm{dpp})\right]$ (25 nm, 0.5:0.5:0.1)/TAPC:Bepp2 $(4 \mathrm{~nm}, 0.7: 0.3) / \mathrm{TPE}-\mathrm{TAPBI}(15 \mathrm{~nm}) / \mathrm{TmPyPB}(40 \mathrm{~nm}) / \mathrm{LiF}(1 \mathrm{~nm}) / \mathrm{Al}$. High singlet energy and triplet energy of the mixed interlayer could effectively prohibit the mutual excitons transfer and quenching processes. The WOLED exhibited warm white light and total power efficiency (PE), current efficiency (CE) and EQE of $70.5 \mathrm{~lm} \mathrm{~W} \mathrm{~W}^{-1}, 76.0 \mathrm{~cd}$ $\mathrm{A}^{-1}$ and $28 \%$ at $1000 \mathrm{~cd} \mathrm{~m}^{-2}$, respectively. The PE, CE and EQE still remained as 49.8 $\operatorname{lm~} \mathrm{W}^{-1}, 75.0 \mathrm{~cd} \mathrm{~A}^{-1}$ and $25.7 \%$ at $5000 \mathrm{~cd} \mathrm{~m}^{-2}$, demonstrating very good efficiency stability (Fig. 1b).

Later, a new blue AIEgen (TPE-TADC) with a higher PLQY of $72.8 \%$ in neat film was prepared by replacing electron-transporting unit PBI with the hole-transporting carbazole groups [31]. The forked carbazole-based substitute could increase carrier transport as well as triplet energy level. TPE-TADC showed a good hole mobility of $7.96 \times 10^{-6} \mathrm{~cm}^{2} \mathrm{~V}^{-1}$ $\mathrm{s}^{-1}$ at an electric field of $5.5 \times 10^{5} \mathrm{~V} \mathrm{~cm}^{-1}$. And the regular horizontal orientation of transition dipole moment of TPE-TADC could enhance the light out-coupling efficiency over 0.3 to contribute to EL efficiency. As expected, the nondoped OLED constructed with TPE-TADC afforded good EL performances of $6.81 \mathrm{~cd} \mathrm{~A}^{-1}, 6.57 \mathrm{~lm} \mathrm{~W}^{-1}$, and 5.71\% (Fig. 1c). By utilizing TPE-TADC as the emissive layer, highly efficient two-color hybrid white OLEDs were achieved, furnishing modulable light color from pure white $\left(\mathrm{CIE}_{\mathrm{x}, \mathrm{y}}=0.33\right.$, $0.33)$ to warm white $\left(\mathrm{CIE}_{\mathrm{x}, \mathrm{y}}=0.44,0.46\right)$ and excellent forward-viewing EL efficiencies of $56.7 \mathrm{~cd} \mathrm{~A}^{-1}, 55.2 \mathrm{~lm} \mathrm{~W} \mathrm{~W}^{-1}$, and 19.2\% (Fig. 1d).

By tuning the twisted angles between the aromatic building blocks, Li et al. [32] successfully developed a benzene-cored blue luminogen 3TPA-CN with aggregationenhanced emission (AEE) property. Based on the excellent hole-transporting ability of 3TPA-CN, the simple nondoped OLED without the hole-transporting layer showed a maximum EQE of 3.89\%. Afterwards, the optimized blue OLED [33] with a structure of ITO/HATCN $(5 \mathrm{~nm}) /$ TAPC $(50 \mathrm{~nm}) /$ TCTA $(5 \mathrm{~nm}) / 3$ TPA-CN $(20 \mathrm{~nm}) /$ TmPyPB $(40$ $\mathrm{nm}) / \mathrm{LiF}(1 \mathrm{~nm}) / \mathrm{Al}$ realized a higher EQE of $6.3 \%$. In addition, 3TPA-CN could also serve as a host material for orange emissive phosphorescent OLED with a maximum EQE of $18.2 \%$, representing that AIEgens had been successfully employed as hosts in phosphorescent OLEDs (PhOLEDs) for the first time. As excellent blue emitter and phosphor host, 3TPA-CN was further used to fabricate two-color WOLED, providing maximum $\mathrm{PE}$ and EQE as high as $86.7 \mathrm{~lm} \mathrm{~W} \mathrm{~m}^{-1}$ and $22.3 \%$, respectively.

Ma et al. [34] developed a multifunctional bipolar deep blue AIEgen, TPB-AC, with a high triplet energy of $2.48 \mathrm{eV}$ and a superior PLQY of $98.6 \%$ in neat film (Fig. 2a). Noticeably, TPB-AC neat film showed a high horizontal dipole ratio of $79 \%$, revealing a great potential in development of high-performance nondoped OLEDs. As a result, TPB-AC was not only suitable to fabricate the pure blue OLED with the maximum EQE of $7.0 \%$ and extremely low efficiency roll-off of $10 \%$ at $1000 \mathrm{~cd} \mathrm{~m}^{-2}$, but also a great host for PhOLEDs with maximum EQEs of $21.0 \%$ for green, $27.3 \%$ for orange, and $26.1 \%$ for red OLEDs, which even still remained at $19.5 \%, 25.9 \%$, and $24.1 \%$ at 


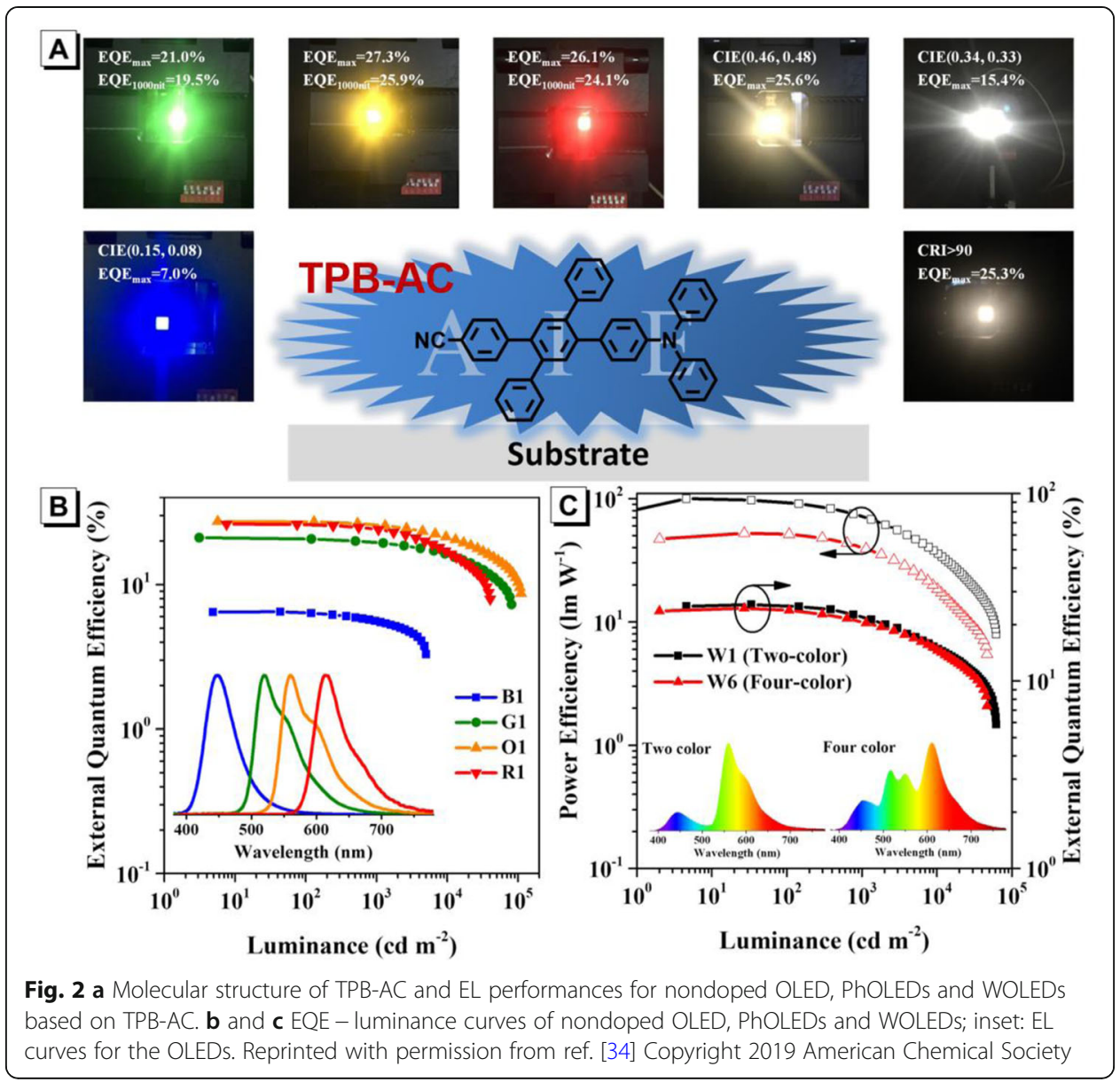

$1000 \mathrm{~cd} \mathrm{~m}^{-2}$, respectively (Fig. 2b). Furthermore, easily manufactured high-efficiency hybrid WOLEDs, which used pure TPB-AC as a blue-emitting layer without existence of interlayer, were fabricated. The maximum CE, PE and EQE reached $83.5 \mathrm{~cd} \mathrm{~A}^{-1}$, $99.9 \mathrm{~lm} \mathrm{~W} \mathrm{~W}^{-1}$ and $25.6 \%$ for the two-color WOLED, and $54.8 \mathrm{~cd} \mathrm{~A}^{-1}, 60.7 \mathrm{~lm} \mathrm{~W}^{-1}$ and $25.3 \%$ for the four-color WOLED, respectively (Fig. 2c).

To increase the efficiency of the OLEDs, it is also a favorable approach to improve $\eta_{\mathrm{r}}$ by harnessing other $75 \%$ triplet excitons through hybridized local and chargetransfer (HLCT) state mechanisms [35, 36]. Wang et al. [37] reported ppCTPI (Fig. 3) based on a typical HLCT core phenanthroimidazole (PI). TPE, a most popular AIE-active building block, was connected to the long axis with para-linkages to tune the locally excited (LE) component in HLCT state (Fig. 3b). Besides, a benzonitrile with a cyano group $(\mathrm{CN})$ was introduced into the short axis to tune the charge transfer (CT) component in HLCT state. Therefore, the "hot exciton" conversion channels between $\mathrm{S}_{1}$ and $\mathrm{T}_{4}\left(\mathrm{~T}_{\mathrm{HLCT}}-\mathrm{S}_{\mathrm{HLCT}}\right)$ as well as $\mathrm{S}_{2}$ and $\mathrm{T}_{8}$ $\left(\mathrm{T}_{\text {HLCT }}-\mathrm{S}_{\mathrm{HLCT}}\right)$ were constructed and conductive to generating more emissive singlet excitons. The nondoped sky-blue OLED with a device configuration of ITO/ HATCN $(5 \mathrm{~nm}) / \mathrm{TAPC}(40 \mathrm{~nm}) / \mathrm{TCTA}(5 \mathrm{~nm}) / p p$ CTPI $(20 \mathrm{~nm}) / \mathrm{TmPyPB}(40 \mathrm{~nm}) /$ LiF $(1 \mathrm{~nm}) / \mathrm{Al}(120 \mathrm{~nm})$ exhibited the maximum luminance, CE, PE and EQE of 31, $070 \mathrm{~cd} \mathrm{~m}^{-2}, 18.46 \mathrm{~cd} \mathrm{~A}^{-1}, 16.32 \mathrm{~lm} \mathrm{~W}^{-1}$ and $7.16 \%$, respectively, with small efficiency roll-off of $4.0 \%$ at $1000 \mathrm{~cd} \mathrm{~m}^{-2}$. 


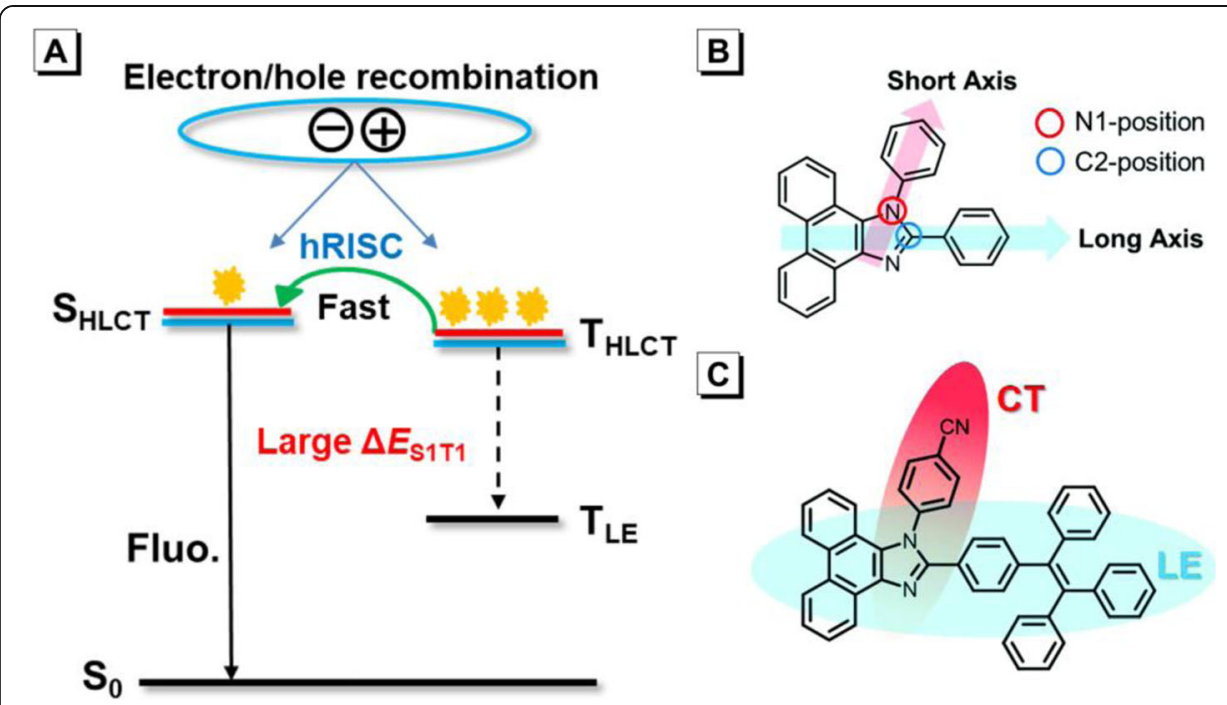

Fig. 3 a Typical energy level characteristics of a HLCT emitter with a "hot exciton" channel; b the long-short axis design based on phenanthroimidazole. c Construction of the LE state and the CT state in ppCTPI. Reprinted with permission from ref. [37] Copyright 2019 Royal Society of Chemistry

In addition, triplet-triplet fusion (TTF) [38, 39] is also feasible to use triplet excitons, in which two triplet excited states can produce one singlet excited state and one ground state, achieving up to $62.5 \%$ exciton utilization (Fig. 4a). Xiao et al. [40] prepared a blue fluorescent emitter TPEA consisting of TPE and anthracenyl, which were directly connected to decrease the conjugation (Fig. 4b). The donor-acceptor (D-A) structure was constructed by introducing weak methoxy donor and cyano acceptor to improve charge balance as well as reduce bathochromic shift by weak intramolecular charge transfer (ICT). No typical $\pi-\pi$ intermolecular interactions were found in crystal, which effectively prevented bathochromic shift to attain deep-blue emission. The PLQY of TPEA was only $4 \%$ in THF but $60 \%$ in nondoped film, showing typical AIE

A

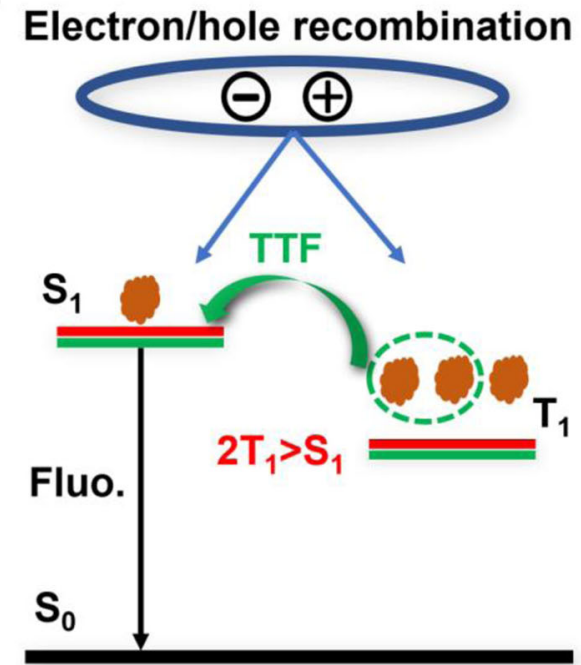

B

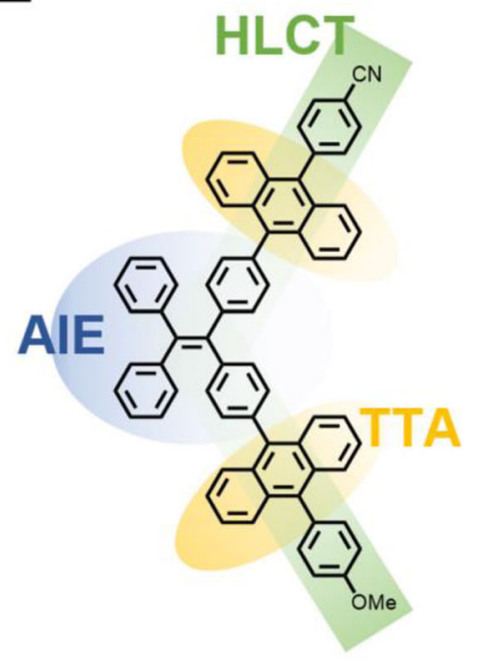

Fig. 4 a Typical energy level characteristic of a TTF emitter. b Molecule structure of TPEA. Reprinted with permission from ref. [40] Copyright 2018 Royal Society of Chemistry 
characteristic. The delayed EL component was observed in time-resolved EL response analysis, confirming the existence of TTF processes. Nondoped blue OLED with TPEA exhibited the maximum PE of $11.1 \mathrm{~lm} \mathrm{~W}^{-1}, \mathrm{CE}$ of $9.9 \mathrm{~cd} \mathrm{~A}^{-1}$, and EQE of $5.6 \%$. To facilitate injection and transport of carriers, broaden the exciton recombination zone and alleviate exciton quenching effect, the doped device was fabricated and displayed deepblue emission at $445 \mathrm{~nm}\left(\mathrm{CIE}_{\mathrm{x}, \mathrm{y}}=0.15,0.09\right)$ with an excellent maximum EQE of 8.0\%.

Host materials play an important role in improving carrier injection and transport, and modulating EL color via energy-transfer mechanism in OLEDs [41-43]. Thus, development of qualitative host materials is of great significance for optimizing EL performance. The bipolar host material, DPMBP-DMAC [44], was designed and behaved with excellent carrier injection and transport ability due to pyridine and 9,9-dimethyl-9, 10-dihydroacridine (DMAC) moieties. The existence of AIE characteristic of host materials could effectively restrain exciton annihilation in light-emitting layer and lower efficiency roll-off of the devices. Highly twisted molecular conformation of DPMBPDMAC could accomplish the separation of highest occupied molecular orbital (HOMO) and lowest unoccupied molecular orbital (LUMO), in favor of bipolar carrier transport and hindrance of exciton quenching in EL (Fig. 5). Several monochromatic devices with different phosphorescent dopants including blue-emissive FIrpic, greenemissive Ir ( $\mathrm{ppy})_{2}(\mathrm{acac})$ and red-emissive Ir ( $\left.\mathrm{piq}\right)_{2}(\mathrm{acac})$ were achieved at very low turnon voltages $(3.1-3.7 \mathrm{~V})$. The maximum EQEs for blue, green, and red PhOLEDs reached $25.12 \%, 24.73 \%$ and $19.71 \%$, respectively, with negligible efficiency roll-off.

On the basis of efficient energy transfer process between the host and guest materials, sufficient utilization of triplet excitons generated in host materials is promising to improve OLED performance [45]. Hence, Li et al. [46] reported the doped polymer lightemitting diodes (PLEDs) with a bipolar TADF host. The $\mathrm{A}_{4}+\mathrm{B}_{2}$ type hyperbranched

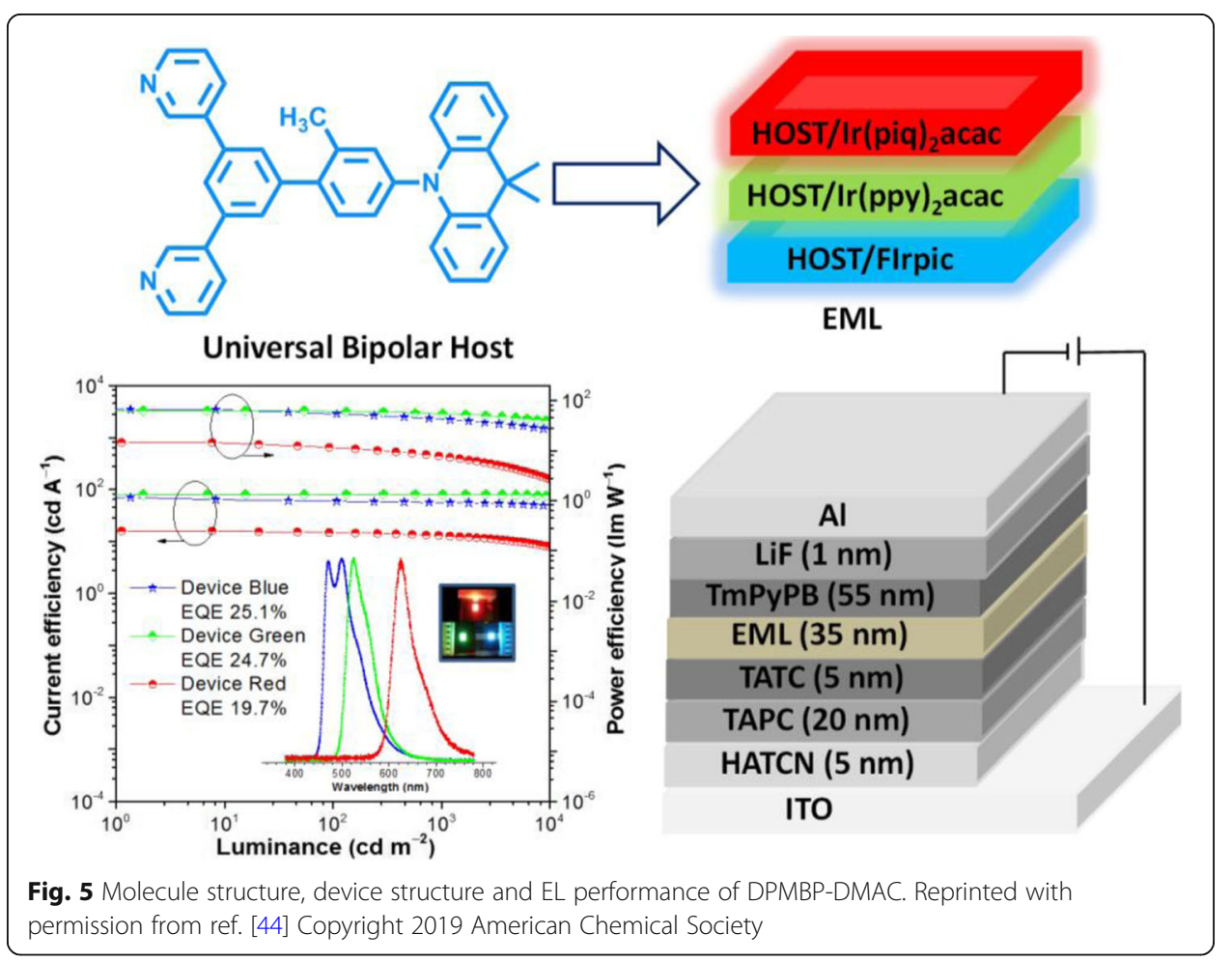


polymer (CP1) of the cyclopentadiene $(\mathrm{CP})$ derivative decorated with TPE was developed. CP1 showed obvious AIE property with a high PLQY of $50.1 \%$ in neat film. Considering the sky-blue emission $(479 \mathrm{~nm})$ in the neat film and high triplet energy level $(3.08 \mathrm{eV})$, the deep blue TADF emitter of CzAcSF was chosen as host. As a result, the solution-processed multilayer PLED with a structure of ITO/PEDOT:PSS $(60 \mathrm{~nm}) /$ CzAcSF:CP1 (5 wt\%, $50 \mathrm{~nm}) /($ oxybis-(2,1-phenylene))-bis-(diphenylphosphine oxide) (DPEPO) $(10 \mathrm{~nm}) / \mathrm{TmPyPB}(60 \mathrm{~nm}) /$ lithium quinolin-8-olate $(\mathrm{Liq})(1 \mathrm{~nm}) / \mathrm{Al}$ showed maximum CE, PE and EQE of $16.96 \mathrm{~cd} \mathrm{~A}^{-1}, 10.75 \mathrm{~lm} \mathrm{~W}^{-1}$ and $9.74 \%$, respectively, with CIE coordinates of $(0.17,0.24)$.

\section{Aggregation-induced delayed fluorescence materials}

Looking through the development history of organic luminescent materials, purely organic thermally activated delayed fluorescence (TADF) [47-49] materials are amongst the most promising candidates in fabricating highly efficient OLEDs. Metal-free TADF can efficiently harvest both singlet and triplet excitons through reverse intersystem crossing (RISC) process, relying on the small singlet-triplet energy gap $\left(\Delta E_{\mathrm{ST}}\right)$ [50-52]. Ideally, the OLEDs based on TADF materials can achieve 100\% internal quantum efficiency, and thus provide excellent EL performances, but there are still some challenges that greatly delay the practical commercial applications of TADF materials. Conventional TADF materials suffer from concentration-caused emission quenching and serious exciton annihilation processes, and thus exhibit drastic efficiency roll-off at high voltages. The integration of AIE property into TADF emitters will effectively alleviate this problem. Up to now, a series of robust aggregation-induced delayed fluorescence (AIDF) luminogens have been developed and brought about a new breakthrough for excellent nondoped OLEDs.

Yasuda et al. [53] developed three AIDF molecules (PCZ-CB-TRZ, TPA-CB-TRZ, and 2PCZ-CB) (Fig. 6) based on electron-deficient icosahedral boron cluster, $o$-carborane, which exhibited strong yellow-to-red emissions with high PLQYs of up to $97 \%$ in neat film. More specifically, the calculated $\Delta E_{\mathrm{ST}}$ values $(0.003-0.146 \mathrm{eV})$ were small enough to achieve effective RISC to facilitate TADF owing to a large spatial separation of the frontier orbitals. The nondoped OLED with a structure of ITO/4,4'-bis-[N-(1naphthyl)- $N$-phenylamino]-1,1'-biphenyl ( $\alpha$-NPD) $(35 \mathrm{~nm}$ )/1,3-bis (carbazol-9-yl) benzene $\quad(\mathrm{mCP}) \quad(10 \mathrm{~nm}) / \mathrm{PCZ}-\mathrm{CB}-\mathrm{TRZ} \quad(20 \mathrm{~nm}) / 2,8$-bis(diphenylphosphoryl)dibenzo[b, d]thiophene) (PPT) $(40 \mathrm{~nm}) / \mathrm{LiF}(0.8 \mathrm{~nm}) / \mathrm{Al}$ displayed an EL peak at $586 \mathrm{~nm}$ with maximum EQE and luminance of $11.0 \%$ and $4530 \mathrm{~cd} \mathrm{~m}^{-2}$, respectively. Besides, other AIDF molecules, PTZ-XT and PTZ-BP [54], were reported by the same group, in which phenothiazine was coupled with xanthone or benzophenone acceptor unit to create DA structures. The maximum EQE values of the nondoped yellow OLEDs based on PTZ-XT and PTZ-BP were $11.1 \%$ and $7.6 \%$, respectively.

Although the EL performances of the above discussed AIDF materials achieved limited advancement, the further works demonstrated that the AIDF materials were indeed promising to break the limit of exciton utilization and alleviate efficiency roll-off. For example, DBT-BZ-DMAC [55] with an asymmetrical D-A-D' structure possessed a prominent AIDF characteristic. The practically perpendicular connection pattern between DMAC and benzoyl moieties with a dihedral angle as large as $87^{\circ}$ was favored to 


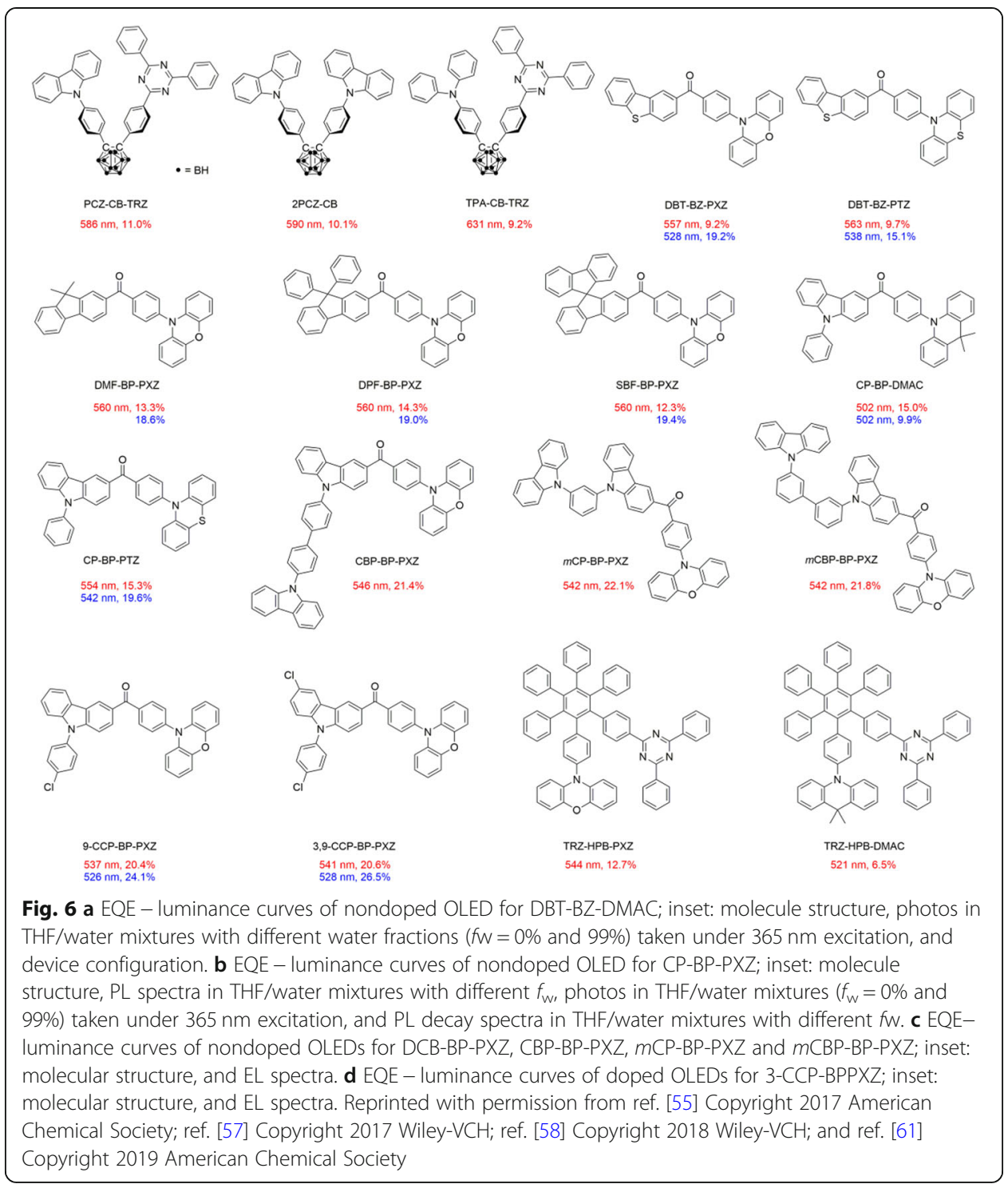

lower the electronic coupling, and thus a small $\Delta E_{\mathrm{ST}}$ of $0.08 \mathrm{eV}$ was attained. DBT-BZDMAC showed a high PLQY of up to $80.2 \%$ in neat film, approximately tenfold higher than that in THF solution. At ambient temperature, the lifetime of delayed fluorescence of the neat film $(2.9 \mu \mathrm{s})$ was much shorter than those of doped films, which was conducive to suppressing efficiency roll-off in nondoped OLEDs. As expected, the nondoped OLED with a configuration of ITO/TAPC $(25 \mathrm{~nm}) /$ DBT-BZ-DMAC $(35 \mathrm{~nm}) /$ TmPyPB $(55 \mathrm{~nm}) / \mathrm{LiF}(1 \mathrm{~nm}) / \mathrm{Al}$ furnished good EL efficiencies of $43.3 \mathrm{~cd} \mathrm{~A}^{-1}, 35.7 \mathrm{~lm} \mathrm{~W} \mathrm{~m}^{-1}$, and $14.2 \%$, with negligible roll-off of $0.46 \%$ at $1000 \mathrm{~cd} \mathrm{~m}^{-2}$ (Fig. 7a). Furthermore, DBT-BZPXZ and DBT-BZ-PTZ [56] (Fig. 6) were synthesized by altering donor moiety, which showed AIDF features and high PLQYs in the aggregated state. The doped OLED employing DBT-BZ-PXZ achieved excellent EL efficiencies of $19.2 \%, 60.6 \mathrm{~cd} \mathrm{~A}^{-1}$, and $59.2 \mathrm{~lm} \mathrm{~W}^{-1}$ and the nondoped device of DBT-BZ-PTZ afforded maximum EL efficiencies of $9.7 \%, 26.5 \mathrm{~cd} \mathrm{~A}^{-1}$, and $29.1 \mathrm{~lm} \mathrm{W^{-1 }}$. 

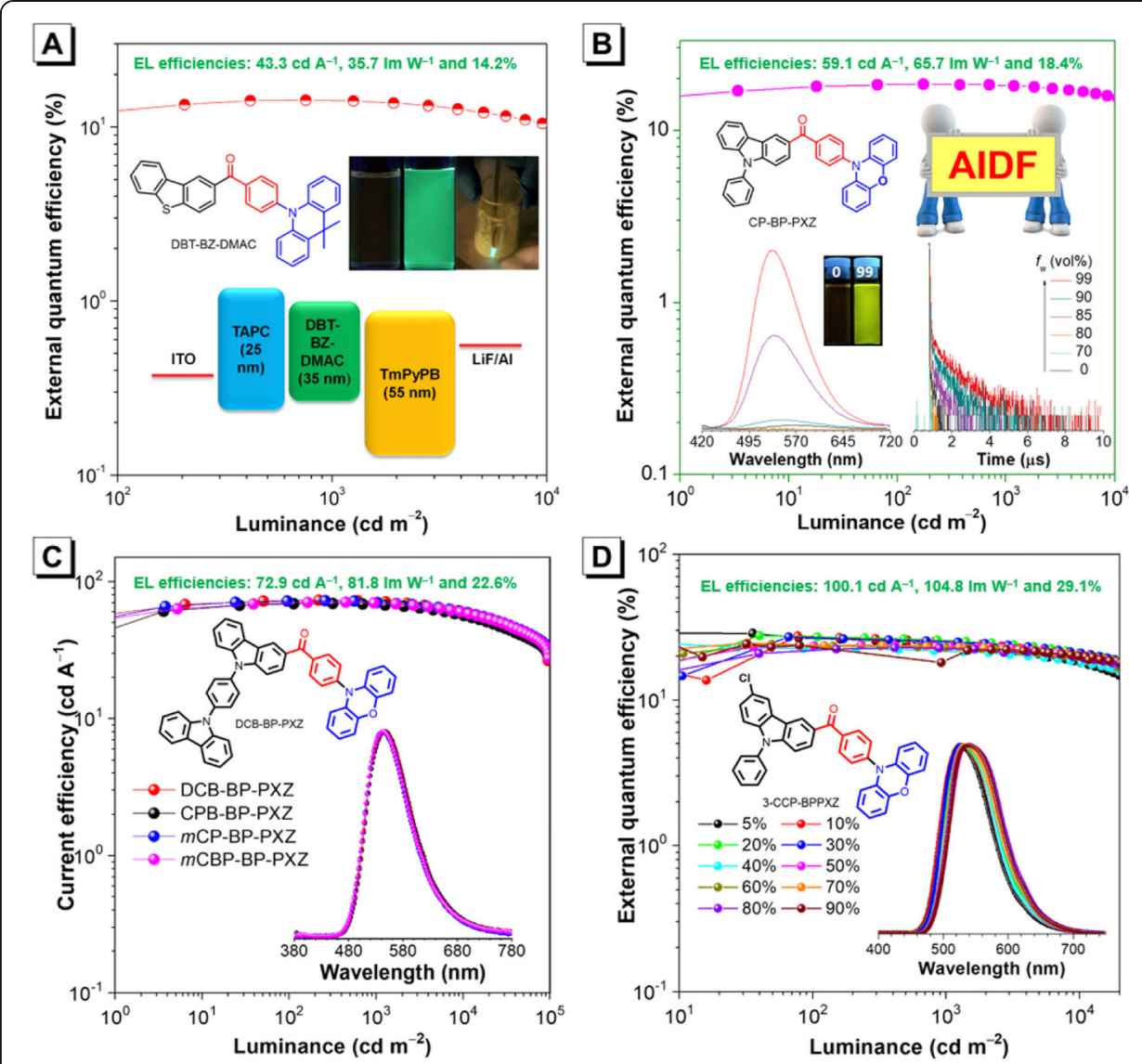

Fig. 7 The main exciton dynamic processes for (a) a conventional TADF emitter and (b) an AIDF emitter in nondoped OLEDs. Reprinted with permission from ref. [57] Copyright 2017 Wiley-VCH

Based on the similar design principle of constructing twisted conformations, it is easy to envision that there should be internal connections between AIE and TADF. Then, the novel AIDF luminogens of CP-BP-PXZ, CP-BP-PTZ, and CP-BP-DMAC (Figs. 6 and $7 \mathrm{~b}$ ) were prepared and studied to deeply investigate the correlation between AIE and TADF [57]. In solution, the intramolecular motions were active and then nonradiative decay of the excited state occurred via fast internal conversion (IC). Consequently, the delayed fluorescence of CP-BP-PXZ was negligible in solution and the average fluorescence lifetime was only $11.8 \mathrm{~ns}$. While in neat film, the intramolecular motions were restricted, and the IC channel was blocked (Fig. 8). Therefore, under the premise of a small $\Delta E_{\mathrm{ST}}$, the intersystem crossing (ISC) and RISC processes became dominative. The delayed fluorescence lifetime was obviously increased to $2.1 \mu$ s for CP-BP-PXZ. The nondoped OLED of CP-BP-PXZ with a structure of ITO/TAPC $(25 \mathrm{~nm}) / \mathrm{emitter}$ $(35 \mathrm{~nm}) / \mathrm{TmPyPB}(55 \mathrm{~nm}) / \mathrm{LiF}(1 \mathrm{~nm}) / \mathrm{Al}$ showed remarkably high EL efficiencies of

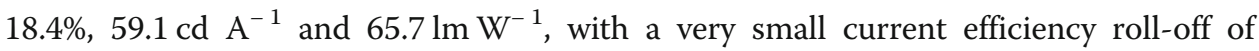
$1.2 \%$ at a luminance of $1000 \mathrm{~cd} \mathrm{~m}^{-2}$ (Fig. 7b). The doped OLED of CP-BP-PTZ also exhibited excellent performance with maximum EL efficiencies of $22.7 \%, 73.3 \mathrm{~cd} \mathrm{~A}^{-1}$ and $69.6 \mathrm{~lm} \mathrm{~W} \mathrm{~W}^{-1}$.

As discussed above, AIDF materials had exhibited impressive merits for fabricating highly efficient nondoped OLEDs. The underlying mechanism of AIDF effect needed to 


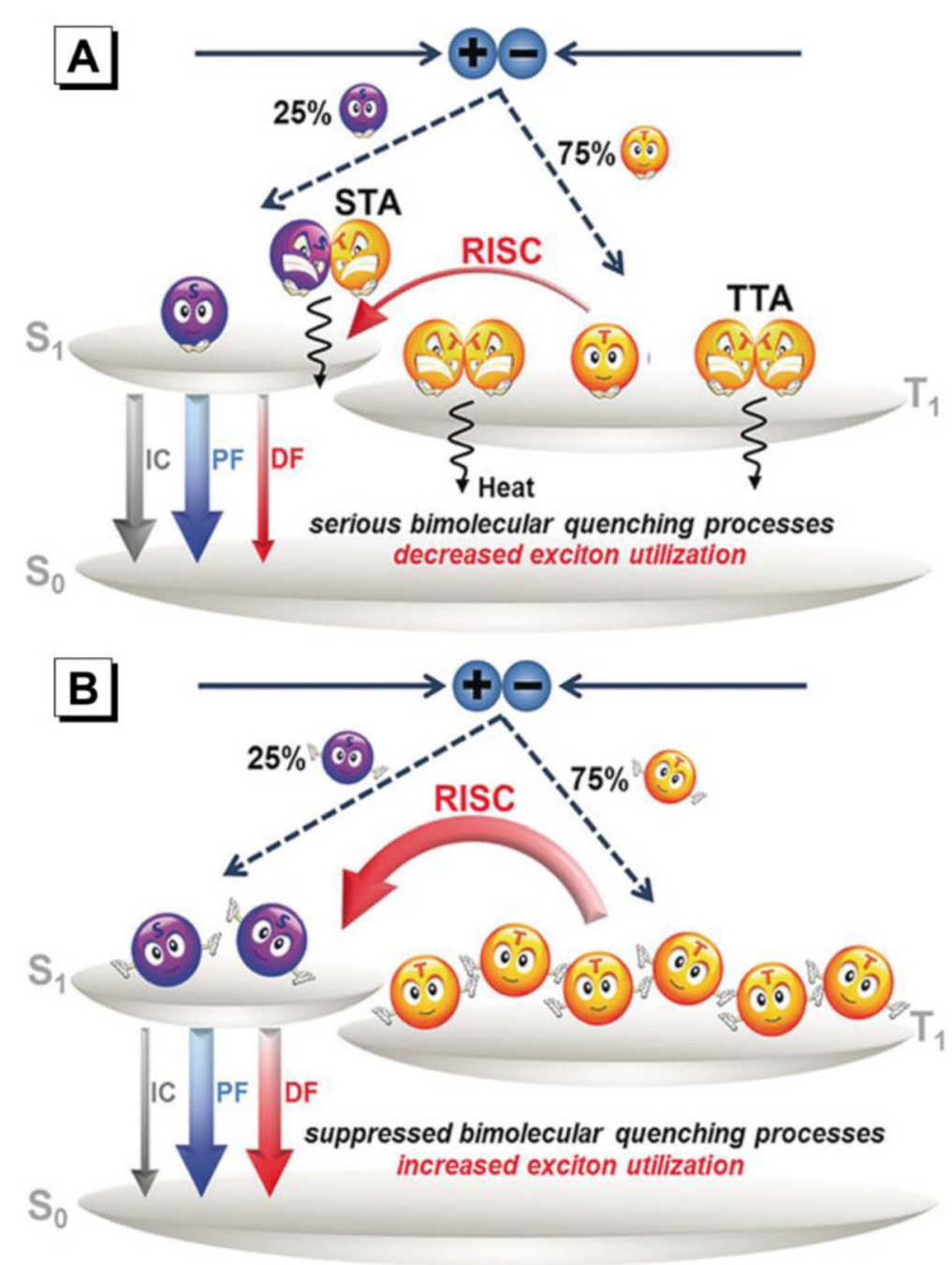

Fig. 8 The proposed photophysical mechanisms for AIDF molecules in solution and solid state. Reprinted with permission from ref. [58] Copyright 2018 Wiley-VCH

be further deciphered for the development more excellent emitters. Then, three new molecules, DMF-BP-PXZ, DPF-BP-PXZ and SBF-BP-PXZ [58] (Fig. 6), were synthesized to study the AIDF process. These materials showed weak emissions with short fluorescence lifetimes in THF solutions. But they could emit strongly and the fluorescence lifetime became much longer with obvious delayed component in neat films (1.1-1.4 $\mu \mathrm{s})$, exhibiting intriguing AIDF properties. By further calculating the photophysical transition rates of these luminogens, it was found that the $k_{\mathrm{IC}}$ values were decreased from solutions $(3.1 \times$ $\left.10^{8}-5.5 \times 10^{8} \mathrm{~s}^{-1}\right)$ to neat films $\left(1.3 \times 10^{7}-1.6 \times 10^{7} \mathrm{~s}^{-1}\right)$, which were comparable to the $k_{\text {ISC }}$ values $\left(7.7 \times 10^{6}-9.7 \times 10^{6} \mathrm{~s}^{-1}\right)$. The highly active intramolecular motions in solutions could act as the rapid IC channels to deactivate the excited state. Consequently, the ISC and RISC processes could hardly occur and the delayed fluorescence disappeared. However, the restriction of intramolecular motions blocked the nonradiative IC channels in the aggregated state, and the ISC and RISC processes were allowed, in favor of the prominent delayed fluorescence under the premise of small singlet-triplet energy splitting (Fig. 9). And the theoretical calculation results further confirmed the dynamical 


\section{Solution}

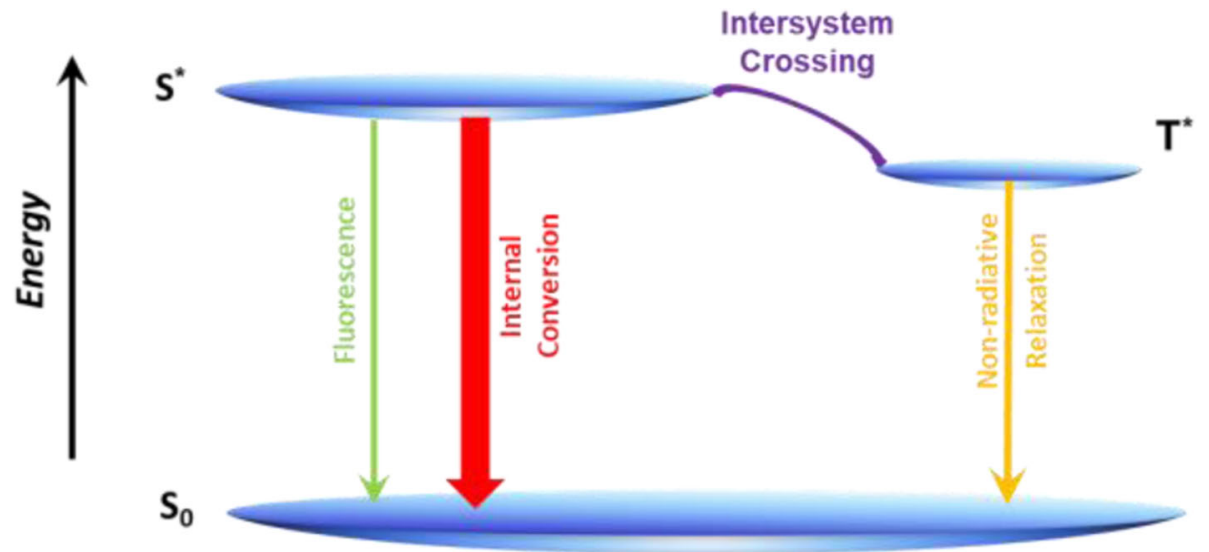

Solid

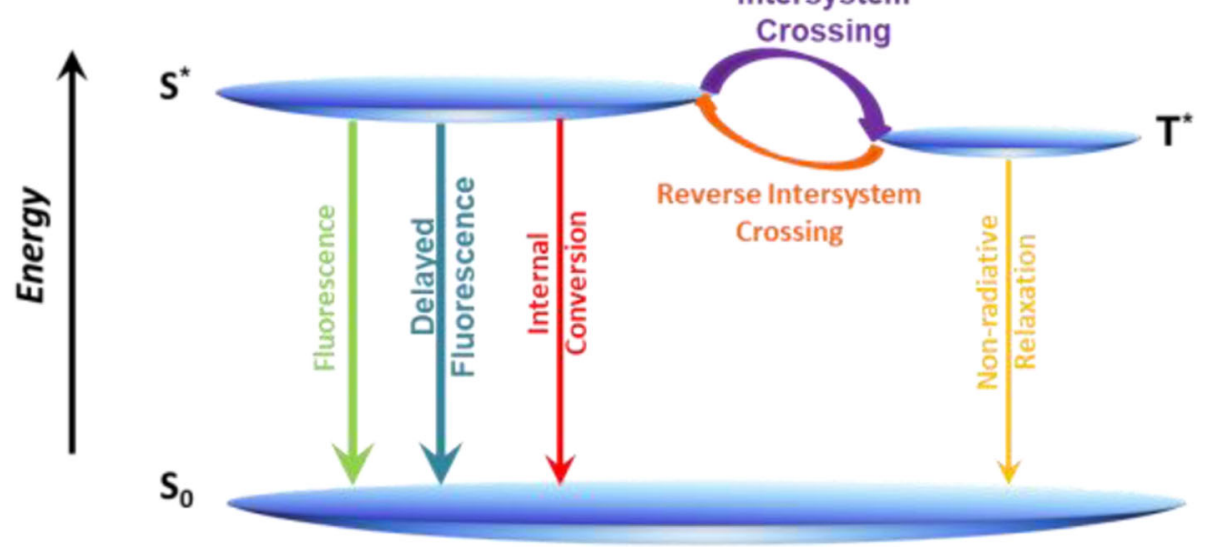

\section{Aggregation-Induced Delayed Fluorescence}

Fig. 9 Molecular structures of delayed fluorescence materials with aggregation-induced emission and EL peaks, maximum EQE of their nondoped (red) and doped (blue) OLEDs

mechanism of AIDF phenomenon. Owing to the unique AIDF property, the EL performance in nondoped and doped OLEDs had also been investigated. The maximum CE, PE and EQE was up to $62.3 \mathrm{~cd} \mathrm{~A}^{-1}, 62.9 \mathrm{~lm} \mathrm{~W}^{-1}$, and $19.4 \%$, respectively, with extremely small efficiency roll-off.

To further improve exciton recombination in emitting layers, host materials were integrated into AIDF molecules to optimize OLED performance. Common host materials were used to synthesize new AIDF materials DCB-BP-PXZ, CBP-BP-PXZ, $m$ CP-BPPXZ and $m$ CBP-BP-PXZ (Figs. 6 and 9) [59]. Crystal structure of CBP-BP-PXZ showed that the phenyl ring was attached to 10-position of PXZ by adopting a large angle of $69.5^{\circ}$, and the phenyl ring of 4,4' -bis(carbazol-9-yl)biphenyl (CBP) moiety also exhibited a highly twisted conformation, with larger torsion angles of $87.5^{\circ}$ and $58.5^{\circ}$. The highly twisted molecular geometry could effectively depress nonradiative decay and inhibit short-range Dexter energy transfer of excitons located at the central benzoyl part. Consequently, the high-concentration excitons at high voltage could be fully utilized 
for emission without severe annihilation in neat films. Nondoped OLEDs with a simple three-layer configuration of ITO/TAPC $(25 \mathrm{~nm}) /$ emitter $(35 \mathrm{~nm}) / \mathrm{TmPyPB}(55 \mathrm{~nm}) / \mathrm{LiF}$ $(1 \mathrm{~nm}) / \mathrm{Al}$ were fabricated. Impressively, the highest luminance of $100,126 \mathrm{~cd} \mathrm{~m}^{-2}$ was achieved in OLED based on $m$ CBP-BP-PXZ. All devices revealed maximum CE, PE and EQE of $69.0-72.9 \mathrm{~cd} \mathrm{~A}^{-1}, 75.0-81.8 \mathrm{~lm} \mathrm{~W}^{-1}$ and 21.4-22.6\%, respectively, with small efficiency roll-off of $8.7-11.4 \%$ at $5000 \mathrm{~cd} \mathrm{~m}^{-2}$ (Fig. 7c).

Theoretically, the short delayed fluorescence lifetime can effectively reduce triplet exciton annihilation at high voltages and alleviate the roll-off of EL efficiency. The heavy atom effect can be strategically utilized to increase the spin-orbit coupling (SOC), realizing a fast RISC and thus a short lifetime [60, 61]. Then, three AIDF molecules decorated with chlorine atom(s), 3-CCP-BPPXZ, 9-CCP-BP-PXZ and 3,9-CCP-BP-PXZ [62] (Figs. 6 and $7 d$ ), were explored. Obvious interactions of chlorine and carbonyl with neighboring aromatic moieties were observed in crystals, inducing efficient orbital couplings. As a result, the calculated SOC values of these luminogens boosted from $0.002-$ $0.007 \mathrm{~cm}^{-1}$ in gas phase to $0.234-0.406 \mathrm{~cm}^{-1}$ in solid phase. Furthermore, 3-CCP-BPPXZ, 9-CCP-BP-PXZ and 3,9-CCP-BP-PXZ had relatively short delayed lifetimes of $0.76,0.68$ and $0.42 \mu \mathrm{s}$, corresponding to the $k_{\mathrm{RISC}}$ of $1.73 \times 10^{6}, 1.97 \times 10^{6}$ and $3.10 \times$ $10^{6} \mathrm{~s}^{-1}$, respectively. In addition, very small $\Delta E_{\mathrm{ST}}$ values of $0.016-0.019 \mathrm{eV}$ and high PLQYs of 70.4-73.0\% were attained. The nondoped OLED of 3-CCP-BP-PXZ provided the maximum $\mathrm{CE}, \mathrm{PE}$, and $\mathrm{EQE}$ of up to $76.6 \mathrm{~cd} \mathrm{~A}^{-1}, 75.2 \mathrm{~lm} \mathrm{~W}^{-1}$, and $21.7 \%$, respectively (Fig. 7d). These new AIDF emitters could also be used to fabricate doped OLEDs with a wide doping concentration range of 5-90 wt\%, giving excellent concentrationinsensitive EL performances. The doped OLED of 3-CCP-BP-PXZ with $5 \mathrm{wt} \%$ doping concentration showed impressive maximum CE, PE, and EQE of $100.1 \mathrm{~cd} \mathrm{~A}^{-1}, 104.8$ $\operatorname{lm} \mathrm{W}^{-1}$, and $29.1 \%$, respectively.

Additionally, Su et al. [63] reported two molecules based on benzophenone, CCDC and CCDD. The branched donors, where a carbazole unit was linked with other carbazole or diphenylamine, could increase the PLQY and the electron donating ability. The PLQY of CCDD with a rotatable structure increased from lower than 1\% in THF solution to $30.8 \%$ in neat film, demonstrating the AIE effect. In contrast, the interlocked phenyl rings on the carbazole induced a coplanar conformation for CCDC (Fig. 10a). The $\Delta E_{\mathrm{ST}}$ of CCDC was $0.147 \mathrm{eV}$ and that of CCDD was $0.045 \mathrm{eV}$. The maximum EQEs of CCDD based nondoped and doped OLEDs were $12.7 \%$ and $22.7 \%$, respectively.

To further enhance solid-state emission efficiency and improve color purity, Cheng et al. [64] designed two new emitters, 2QPM-mDTC and 4QPM-mDTC, based on the benzophenone acceptor (Fig. 10b). The introduction of a secondary acceptor, quinoline, in 2QPM-mDTC could induce intramolecular hydrogen bonding between the $o$-hydrogen on the central phenyl and the nitrogen of quinoline. Therefore, the molecular structure of 2QPM-mDTC was rigidified to certain degree, which was beneficial to narrow emission peak and enhance emission intensity. The PLQY of 2QPM-mDTC was up to $98 \%$ in 7 wt\% doped 3,3' -di(carbazol-9-yl)biphenyl (mCBP) film, which was much higher than that in toluene (6.5\%), presenting obvious AIE property. On the contrary, the hydrogen bonding couldn't form in 4QPM-mDTC because of the long distance between nitrogen and $o$-hydrogen, resulting in a relatively lower PLQY of $80 \%$ in doped film. In addition, the RISC between singlet charge transfer $\left({ }^{1} \mathrm{CT}\right)$ state and triplet local excited ( $\left.{ }^{3} \mathrm{LE}\right)$ state was favored in these two molecules, leading to delayed fluorescence. 


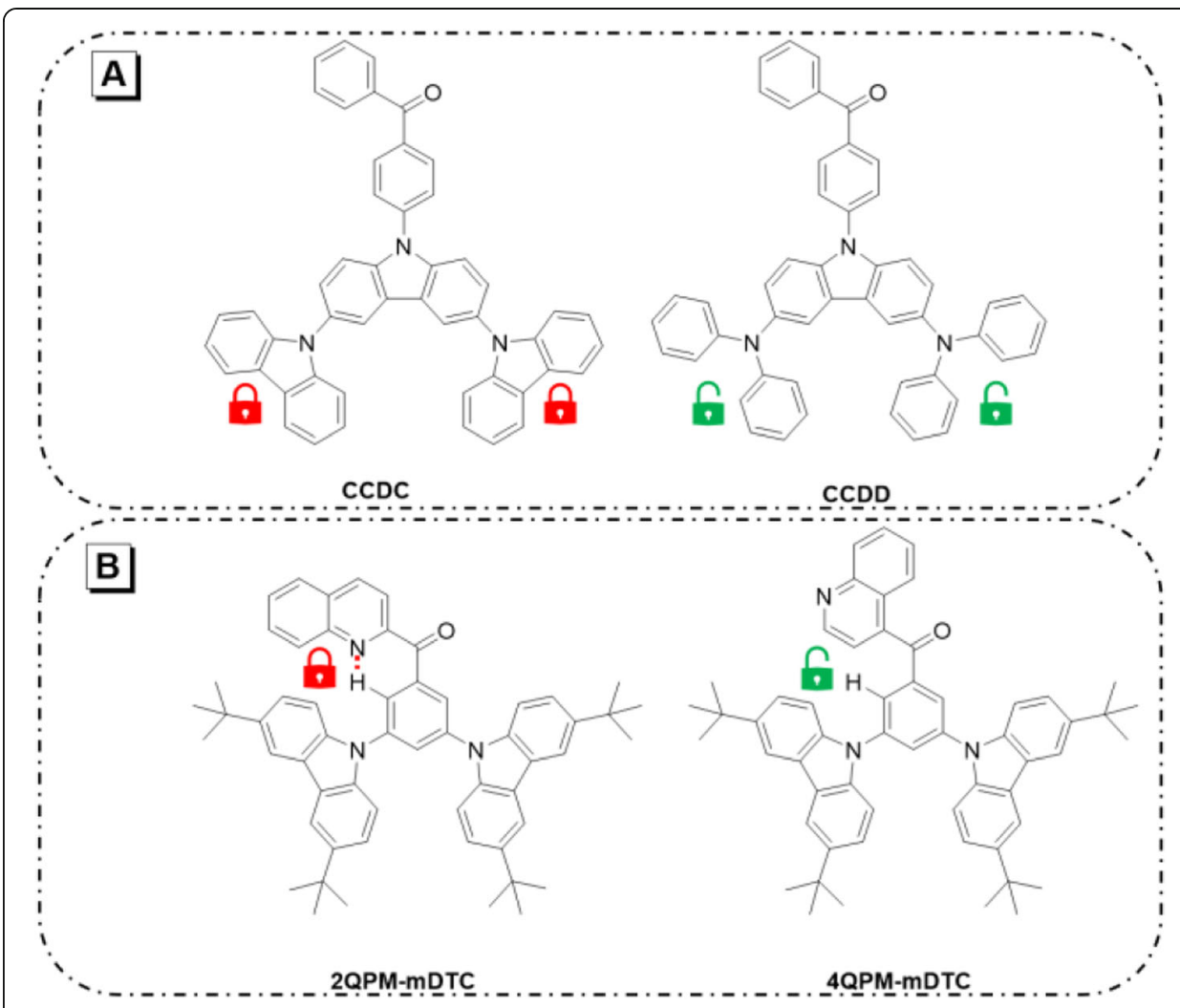

Fig. 10 Molecular structures of (A) CCDC and CCDD, and (B) 2QPM-mDTC and 4QPM-mDTC. Reprinted with permission from ref. [63] Copyright 2017 Royal Society of Chemistry and Chinese Chemical Society

The doped OLED fabricated by 2QPM-mDTC could afford high maximum EQE, CE and $\mathrm{PE}$ of $24.0 \%, 79.5 \mathrm{~cd} \mathrm{~A}^{-1}$ and $62.3 \mathrm{~lm} \mathrm{~W}^{-1}$, respectively.

Wang et al. [65] developed a novel acridine-carbazole hybrid donor to construct efficient emitters 34AcCz-PM and 34AcCz-Trz. TADF and AIE features were simultaneously integrated into these two emitters with highly twisted structures. Multiple intermolecular interactions between donor and acceptor moieties were found, which were beneficial for the improvement of molecular rigidification and reduction of nonradiative radiative channels. As a result, a high PLQY of up to $67 \%$ was achieved in neat film. The maximum $\mathrm{CE}, \mathrm{PE}$ and EQE of $73.3 \mathrm{~cd} \mathrm{~A}^{-1}, 72.4 \mathrm{~lm} \mathrm{~W} \mathrm{~W}^{-1}$ and $22.6 \%$ were obtained for the doped OLED based on $34 \mathrm{AcCz}-\mathrm{PM}$, which remained at $65.5 \mathrm{~cd} \mathrm{~A}^{-1}, 27.9 \mathrm{~lm} \mathrm{~W} \mathrm{~m}^{-1}$ and $20.1 \%$ even at a high luminance of 5000 $\mathrm{cd} \mathrm{m}^{-2}$. Nondoped OLED of 34AcCz-PM revealed excellent maximum EL efficiencies of $45.2 \mathrm{~cd} \mathrm{~A}^{-1}, 40.0 \mathrm{~lm} \mathrm{~W}^{-1}$ and $14.1 \%$. The same group also reported another AIDF emitter, PXZ2PTO [66], consisting of a novel electron acceptor based on 10phenyl-10H-phenothiazine 5,5-dioxide. In crystal structure, molecular packing pattern occupied an obvious $\pi-\pi$ stacking between phenoxazine units of adjacent molecules with a distance of $3.608 \AA$, which was in favor of charge transport. Multiple hydrogen bonds in the highly twisted conformation could efficiently suppress the nonradiative decay and enhance the emission efficiency. The green nondoped OLED fabricated by PXZ2PTO realized high maximum EQE, CE and PE of 16.4\%, $44.9 \mathrm{~cd} \mathrm{~A} \mathrm{~A}^{-1}$ and $32.0 \mathrm{~lm} \mathrm{W^{-1 }}$, respectively. 
Traditional TADF molecule design strategy normally focuses on creating a twisted structure through direct connection between donor and acceptor, resulting in the difficult balance between small $\Delta E_{\mathrm{ST}}$ and high PLQY. Chi et al. [67] reported an effective structural design strategy by cooperating through-bond charge transfer and throughspace charge transfer in 2Cz-DPS, wherein donor and acceptor groups were linked at the ortho-position to obtain the spatially close D-A interaction (Fig. 11a). The dual charge transfer pathways greatly enhanced the oscillator strength for faster radiative decay, exhibiting a high PLQY of $91.9 \%$ in solid. In addition, the non-adiabatic coupling between the triplet $\mathrm{CT}$ and LE played an important role in enhancing the RISC process (Fig. 11b). Thus, small $\Delta E_{\mathrm{ST}}$, reduced vibrations and suppressed nonradiative pathways accompanied by twisted geometry led to effective AIE and TADF properties. Impressively, the nondoped OLED constructed with a configuration of ITO/PEDOT: PSS (30 $\mathrm{nm}) / \mathrm{mCP}(20 \mathrm{~nm}) / 2 \mathrm{Cz}$-DPS $(30 \mathrm{~nm}) / \mathrm{TPBI}(40 \mathrm{~nm}) / \mathrm{LiF}(1 \mathrm{~nm}) / \mathrm{Al}$ achieved maximum EQE, CE and PE of $28.7 \%, 82.3 \mathrm{~cd} \mathrm{~A}^{-1}$ and $51.7 \mathrm{~lm} \mathrm{~W} \mathrm{~W}^{-1}$, respectively, at a luminance of $10 \mathrm{~cd} \mathrm{~m}^{-2}$. On the basis of this design method, hexaphenylbenzene-based luminogens

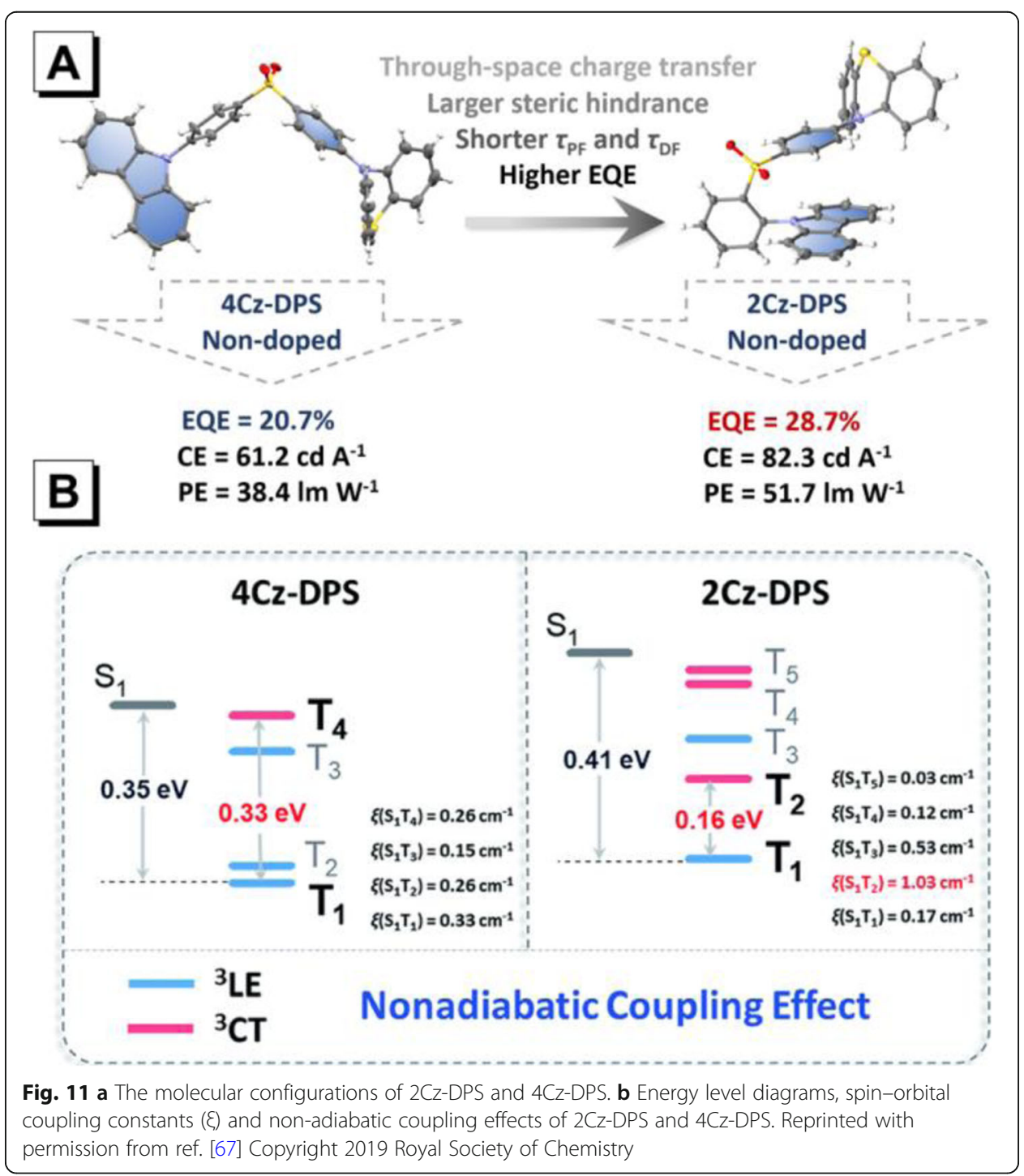


TRZ-HPB-PXZ and TRZ-HPB-DMAC [68] were also created (Fig. 6), in which through-space charge transfer occurred between donor and acceptor moieties, favoring efficient RISC. TRZ-HPB-PXZ and TRZ-HPB-DMAC exhibited high PLQYs of 61.5\% and $51.8 \%$, respectively. The nondoped device of TRZ-HPB-PXZ provided maximum EQE, CE and PE of $12.3 \%, 40.1 \mathrm{~cd} \mathrm{~A}^{-1}$ and $31.5 \mathrm{~lm} \mathrm{~W}^{-1}$, respectively.

Solution-processed nondoped OLEDs with low-cost and high reproducibility possess bright prospects in commercial applications. Yang et al. [69] synthesized two blue emitters, $o$-ACSO2 and $m$-ACSO2, with excellent solubility and AIDF property to fabricate the highly efficient solution-processed nondoped OLEDs. In comparison with $o$-ACSO2, $m$-ACSO2 revealed blue-shifted emission but increased $\Delta E_{\mathrm{ST}}$ due to its weakened conjugation originating from the meta-linkage. The PLQYs of $m$-ACSO2 increased from $69 \%$ in the doped films to $76 \%$ in neat films. Then, the solution-processed nondoped OLED employing $m$-ACSO2 as emitter with an optimized device configuration of ITO/PEDOT: PSS $(60 \mathrm{~nm}) / m-\mathrm{ACSO} 2(60 \mathrm{~nm}) / \mathrm{DPEPO}(10 \mathrm{~nm}) / \mathrm{TmPyPB}(50 \mathrm{~nm}) / \mathrm{Liq}(1 \mathrm{~nm}) / \mathrm{Al}(100$ $\mathrm{nm})$ was fabricated. The nondoped sky-blue device based on $m$-ACSO2 achieved maximum EQE, CE and PE of $17.2 \%, 37.9 \mathrm{~cd} \mathrm{~A}^{-1}$ and $23.8 \mathrm{~lm} \mathrm{~W}^{-1}$, respectively. To increase the film-forming ability of small molecules, long alkyl chains are frequently selected as the functional groups. Two AIDF molecules based on benzophenone, CC6-DBP-PXZ and CC6-DBP-PXZ [70], were reported. The PLQYs of CC6-DBP-PXZ and CC6-DBP-DMAC increased from $4.1 \%$ and $11.7 \%$ in THF to $38.3 \%$ and $59.5 \%$ in neat films, respectively. The CC6-DBP-PXZ-based doped devices showed maximum EQE, CE and PE of 12.1\%, $37.6 \mathrm{~cd} \mathrm{~A}^{-1}$ and $17.8 \mathrm{~lm} \mathrm{~W}^{-1}$, respectively. The CC6-DBP-DMAC-based nondoped devices showed maximum EQE, CE and PE of $9.02 \%, 25.08 \mathrm{~cd} \mathrm{~A}^{-1}$ and $11.25 \mathrm{~lm} \mathrm{~W}^{-1}$ (Fig. 12), respectively, which very small efficiency roll-off.

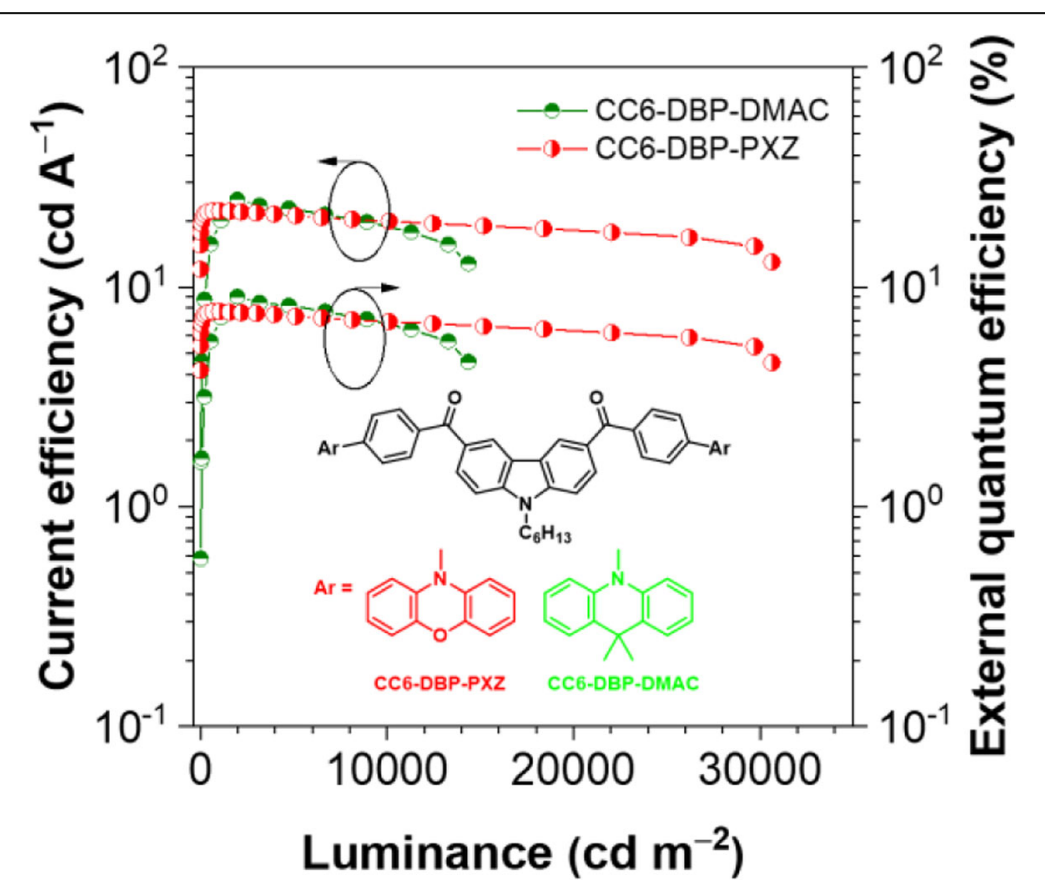

Fig. 12 Current efficiency-luminance-external quantum efficiency characteristics of the nondoped devices. Configurations: ITO/PEDOT:PSS (50 nm)/PVK (30 nm)/emitter/TmPyPB (40 nm)/LiF (1 nm)/Al. Reprinted with permission from ref. [70] Copyright 2019 Royal Society of Chemistry 


\section{AlEgens for circularly polarized luminescence}

Circularly polarized luminescence [71-73] materials have inspired attractive interests for the potential applications, such as chiroptical recognition sensors, bio-responsive imaging and optoelectronic devices. Generally, in OLED displays, antiglare filters are used to remove glare from external light sources by exploiting the nature of circle polarized (CP) light. Meanwhile, nearly $50 \%$ of the nonpolarized light emitted from the OLED pixel is also eliminated, leading to large efficiency loss. In contrast, circularly polarized OLEDs (CPOLEDs) [74] can directly generate CP light with simple device architectures, which will open up a new avenue for organic optoelectronic devices. So far, various kinds of CPL materials have been continuously developed to fabricate CPOLEDs with intense circularly polarized EL. However, it is still challenging to realize highly efficient CPOLEDs due to the obstacle of ACQ problems of the chiral emitting materials. Therefore, the development of effective chiral emitters with AIE effect will be of great significance for future display.

Axial chirality moieties are extensively adopted as blocks to build CPL materials, such as binaphtyl, ctahydro-binaphthol, diaminocyclohexane, and their derivatives. To pursue the CPOLEDs with high EQEs and small efficiency roll-off, a series of CPL emitters with AIE and TADF characteristics $R / S$-BN-CF, $R / S$-BN-CCB, $R / S$-BN-DCB, and $R / S$ BN-AF [75], were developed based on binaphthalene unit (Fig. 13a). The single-crystal structures of $R$-BN-CF and $S$-BN-DCB demonstrated twisted molecular conformations and multiple weak intermolecular interactions, which efficiently suppressed concentration quenching and exciton annihilation. Therefore, the PLQY of $S$-BN-CF in neat film was $38.7 \%$, which fell to $2 \%$ in methanol solution, suggesting the typical AIE feature. In addition, the twisted structure was conductive to low electronic coupling and efficient separation of HOMO and LUMO. Thus, small $\Delta E_{\mathrm{ST}}$ values of $0.02-0.15 \mathrm{eV}$ were obtained, rendering the molecule with prominent delayed fluorescence property. The circularly polarized absorption dissymmetry factors $\left(g_{\text {abs }}\right)$ of these molecules in the longer wavelength region were associated with the absorption transition of $\mathrm{D}-\mathrm{A}$ electronic structures, suggesting the chirality transfer from chiral 1,1'-bi-2-naphthol (BINOL)

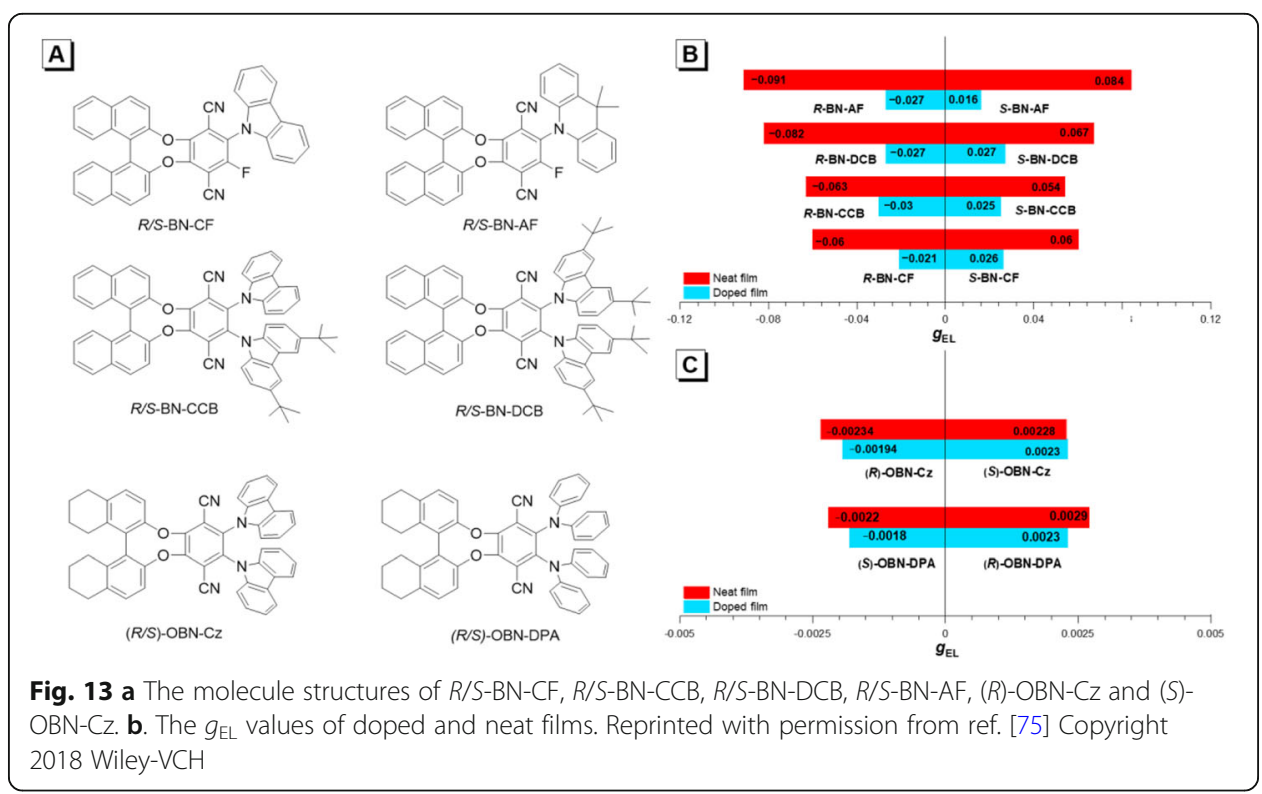


skeleton to the whole conjugated molecules. Moreover, the $g_{\text {abs }}$ and circularly polarized photoluminescence dissymmetry factors $\left(g_{\mathrm{PL}}\right)$ of these molecules were magnified from solution state to film state. In other words, the AIEgens tended to form the population of chiral aggregates in cluster process, which indicated the chiroptical amplification of both ground state and excited state. The configuration of nondoped OLED was illustrated as ITO/HATCN $(10 \mathrm{~nm}) /$ TAPC $(60 \mathrm{~nm}) / \mathrm{mCP}(10 \mathrm{~nm}) / S$-BN-CF $(20 \mathrm{~nm}) /\left(3,3^{\prime \prime}\right.$, 5,5" -tetra(pyridin-3-yl)-1,1': $3^{\prime}, 1^{\prime \prime}$-terphenyl) (BmPyPB) $(50 \mathrm{~nm}) / \mathrm{Liq}(2.5 \mathrm{~nm}) / \mathrm{Al}$. The maximum luminance, $\mathrm{CE}$ and $\mathrm{EQE}$ of $2570 \mathrm{~cd} \mathrm{~m}^{-2}, 10.28 \mathrm{~cd} \mathrm{~A}^{-1}$ and $3.51 \%$, respectively, with circularly polarized EL dissymmetry factors $\left(g_{\mathrm{EL}}\right)$ value of +0.026 were attained. The doped device of $S$-BN-CF exhibited a $g_{\mathrm{EL}}$ value of +0.06 with the maximum luminance, $\mathrm{CE}$ and $\mathrm{EQE}$ of $2948 \mathrm{~cd} \mathrm{~m}^{-2}, 24.58 \mathrm{~cd} \mathrm{~A}^{-1}$ and $9.31 \%$, respectively. The high $g_{\mathrm{EL}}$ for nondoped device may be attributed to the population of chiral aggregates in neat film (Fig. 13b).

Zheng et al. [76] reported a pair of TADF enantiomers $(R)-\mathrm{OBN}-\mathrm{Cz}$ and $(S)-\mathrm{OBN}-\mathrm{Cz}$ (Fig. 13a), in which the octahydro-binaphthol (OBN) units served as chiral sources to generate and induce chirality. Besides, due to peripheral sixteen hydrogen atoms in cyclohexane parts, OBN unit possessed great steric hindrance to suppress close stacking of molecules. For $(R)-\mathrm{OBN}-\mathrm{Cz}$, the neat film and doped film showed higher PLQYs of $81 \%$ and $92 \%$ than those in toluene, demonstrating obvious AIE effect. In addition, the OBN units also could induce a regular orientation to generate horizontal dipoles in the vacuum-evaporating process for increasing the light out-coupling of OLEDs. The TADF property was introduced by the interaction of carbazole and cyan moieties, which were occupied by HOMO and LUMO, respectively. As a result, the small experimental $\Delta E_{\mathrm{ST}}$ of $0.037 \mathrm{eV}$ for $(R)-\mathrm{OBN}-\mathrm{Cz}$ led to efficient RISC process. The maximum band around $400 \mathrm{~nm}$ could be allocated to the absorption of the D-A electronic structure, induced by chirality of OBN unit in the ground state. The CPL spectra of enantiomers showed $g_{\text {PL }}$ values of $-4.6 \times 10^{-4}$ for $(R)$-OBN-Cz and $+5.6 \times 10^{-4}$ for $(S)$-OBN$\mathrm{Cz}$ in toluene. Whereas the $g_{\mathrm{PL}}$ values of enantiomers both in neat and doped films were significantly improved to $1.55 \times 10^{-3}$ and $2.14 \times 10^{-3}$, respectively, revealing chirality amplification effect in aggregation process. The CPOLED based on D-D $(R)$ was fabricated as ITO/HATCN $(10 \mathrm{~nm}) / \mathrm{TAPC}(60 \mathrm{~nm}) / \mathrm{TCTA}:(R)-\mathrm{OBN}-\mathrm{Cz}(10 \mathrm{wt} \%)$ (5 $\mathrm{nm}) / 26 \mathrm{DCzPPy}:(R)-\mathrm{OBN}-\mathrm{Cz}(10 \mathrm{wt} \%)(15 \mathrm{~nm}) / \mathrm{TmPyPB}(60 \mathrm{~nm}) / \mathrm{LiF}(1 \mathrm{~nm}) / \mathrm{Al}$. For the double-emitting layer, TCTA could act as both hole-transporting "ladder" and host, which would lower the driving voltage and broad the exciton recombination zone. The device of $\mathrm{D}-\mathrm{D}(R)$ showed green emission at $501 \mathrm{~nm}\left(\mathrm{CIE}_{\mathrm{x}, \mathrm{y}}=0.22,0.53\right)$ with a full width at half maximum (FWHM) of $66 \mathrm{~nm}$. It is noticeable that the device of D-D $(R)$ displayed maximum brightness, $\mathrm{CE}, \mathrm{PE}$ and EQE of 46,651 $\mathrm{cd} \mathrm{m}^{-2}, 93.7 \mathrm{~cd} \mathrm{~A}^{-1}, 59.3$ $\operatorname{lm~} \mathrm{W}^{-1}$ and $32.6 \%$, respectively, with a $g_{\mathrm{EL}}$ value of $-1.94 \times 10^{-3}$. The nondoped device of D-NF $(R)$ showed maximum brightness, CE, PE and EQE of $35,633 \mathrm{~cd} \mathrm{~m}^{-2}, 47.8 \mathrm{~cd}$ $\mathrm{A}^{-1}, 34.6 \mathrm{~lm} \mathrm{~W} \mathrm{~W}^{-1}$ and $14.0 \%$, respectively, with a $g_{\mathrm{EL}}$ value of $-2.34 \times 10^{-3}$ (Fig. 13c). In addition, triphenylamine-substituted enantiomer, (R/S)-OBN-DPA [77], was synthesized by the same group. The nondoped and doped CPOLEDs achieved markedly CPL signals with $g_{\mathrm{EL}}$ values of up to $2.9 \times 10^{-3}$ (Fig. 13c), high brightness over $25,000 \mathrm{~cd} \mathrm{~cm}^{-2}$ and high maximum EQE of $12.4 \%$.

Chen et al. [78] synthesized two enantiomers, (+)-(S,S)-CAI-Cz and (-)-(R,R)-CAI-Cz (Fig. 14), by cooperating chiral unit of 1,2-diaminocyclohexane with TADF skeleton. 


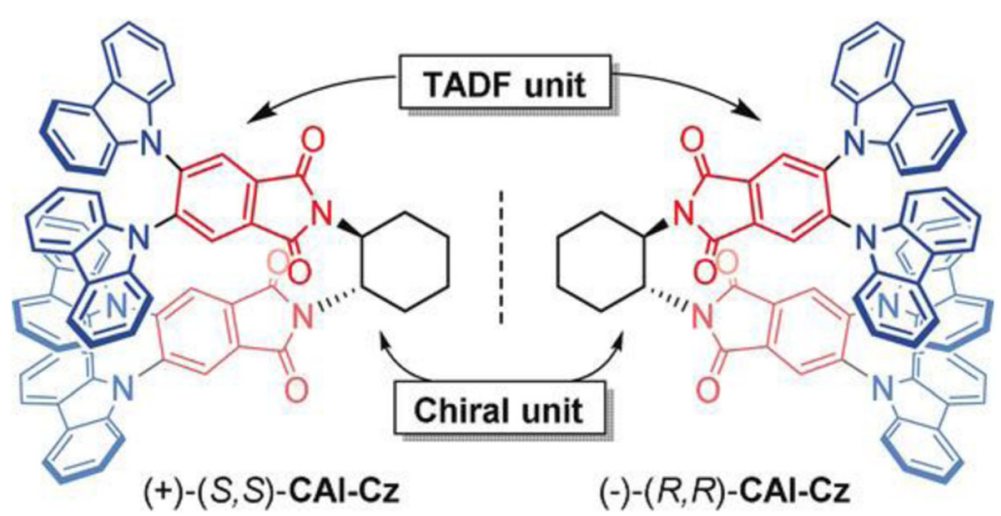

Fig. 14 The molecule structures of $(+)-(S, S)-C A I-C z$ and $(-)-(R, R)-C A I-C z$. Reprinted with permission from ref [78] Copyright 2018 Wiley-VCH

The PLQY of $(+)-(S, S)-C A I-C z$ was $41 \%$ in film and increased to $98 \%$ in mCBP doped film, which was higher than those in solutions. The doped film showed a small $\Delta E_{\mathrm{ST}}$ value of $0.06 \mathrm{eV}$ and thus a fast RISC rate of $2.0 \times 10^{4} \mathrm{~s}^{-1}$, in favor of TADF property. In spite of the nonparticipation of the 1,2-diaminocyclohexane unit in frontier orbitals, it still efficiently induced chiroptical signals of enantiomers as reflected in circular dichroism (CD) and CPL spectra. The nearly mirror-image CPL spectra of the enantiomers were also investigated with $g_{\text {lum }}$ of $-1.1 \times 10^{-3}$ for the $(+)-(S, S)-\mathrm{CAI}-\mathrm{Cz}$ and + $1.1 \times 10^{-3}$ for $(-)-(\mathrm{R}, \mathrm{R})-\mathrm{CAI}-\mathrm{Cz}$. The doped OLED with a structure of ITO/HATCN $(10 \mathrm{~nm}) / \mathrm{TAPC}(25 \mathrm{~nm}) / \mathrm{TCTA}(10 \mathrm{~nm}) / \mathrm{mCBP}(10 \mathrm{~nm}) / \mathrm{mCBP}:$ enantiomers $(15 \mathrm{wt} \%)$ $(20 \mathrm{~nm}) / \mathrm{TmPyPB}(45 \mathrm{~nm}) / \mathrm{Liq}(1 \mathrm{~nm}) / \mathrm{Al}$ was fabricated. The device of $(-)-(R, R)$-CAI$\mathrm{Cz}$ showed a $g_{\mathrm{EL}}$ value of $2.3 \times 10^{-3}$ and emitted green light peaking at $520 \mathrm{~nm}$ with maximum CE, PE and EQE of $59.4 \mathrm{~cd} \mathrm{~A}^{-1}, 52.9 \mathrm{~lm} \mathrm{~W}^{-1}$ and $19.8 \%$, respectively.

Cheng et al. [79] synthesized chiral BINOL-based chiral polymers, $S$-P and $R-P$, by the Pd-catalyzed Suzuki coupling polymerization reaction. The clear mirror-image $C D$ bands were observed in spin-coated films of $S$-/R-P enantiomers. Moreover, the conjugated structures of two chiral binaphthyl moieties bridged through AIE-active ethenyl linker showed a weak cotton effect peak situated at about $360 \mathrm{~nm}$, indicating that the efficient chirality transfer in the whole polymer chain system. Meanwhile, $S / R-P$ enantiomers emitted CPL emission with a peak at $496 \mathrm{~nm}$ and the $g_{\mathrm{PL}}$ values of $S-\mathrm{P}$ and $R-\mathrm{P}$ corresponded to $+1.1 \times 10^{-3}$ and $-1.3 \times 10^{-3}$, respectively. To evaluate the circularly polarized EL performance of the enantiomers, OLEDs with configurations of ITO/ PEDOT: PSS $(25 \mathrm{~nm}) / S$-P or $R$-P $(45 \mathrm{~nm}) / \mathrm{TPBI}(35 \mathrm{~nm}) / \mathrm{Ca}(10 \mathrm{~nm}) / \mathrm{Ag}$ were fabricated. The maximum CE, PE and brightness were $0.926 / 0.833 \mathrm{~cd} \mathrm{~A}^{-1}, 0.390 / 0.422 \mathrm{~lm} \mathrm{~W}^{-1}$ and $1669 / 1270 \mathrm{~cd} \mathrm{~m}^{-2}$ for the doping-free devices based on $S$-P and $R$-P, respectively. The corresponding $g_{\mathrm{EL}}$ values were evaluated as +0.024 and -0.019 . To further improve the performances of CPOLEDs, the same group was incorporated with TADF mechanism to harvest triplet excitons, and two pairs of small molecules $R-1 / S-1$ and $R$ $2 / S-2$ were designed by extending the conjugated skeletons of chiral BINOL [80]. $R-1 /$ $S-1$ and $R-2 / S-2$ possessed prominent AIE property and displayed emission peaks at 587 and $547 \mathrm{~nm}$ in neat films with PLQYs of $2.3 \%$ and $5.9 \%$, respectively. Meanwhile, the PLQYs were greatly enhanced to $18.5 \%$ and $15.7 \%$ when doping in TCTA. For $S-1$ and $S-2$, the HOMO was distributed on phenoxazine moiety, whereas the LUMO was 
located on chiral benzophenone or xanthenone moieties. The effective separation of HOMO and LUMO was favored to the occurrence of TADF with high $k_{\text {RISC }}$ values of $1.4 \times 10^{6} \mathrm{~s}^{-1}$ and $4.2 \times 10^{6} \mathrm{~s}^{-1}$. By fixing the conjugation skeleton with oxygen bridge, the $g_{\mathrm{PL}}$ values of $R-1$ and $S-1$ were $-1.2 \times 10^{-3} /+1.6 \times 10^{-3},-7.1 \times 10^{-4} /+9.2 \times 10^{-4}$ and $-7.2 \times 10^{-4} /+8.2 \times 10^{-4}$ in toluene, neat films, and doped films, respectively, whereas no CPL signals were detected for $R-2 / S-2$. The doped CPOLED based on $R-1$ provided maximum brightness, $\mathrm{CE}$ and EQE of $40,470 \mathrm{~cd} \mathrm{~m}^{-2}, 9.1 \mathrm{~cd} \mathrm{~A}^{-2}$ and $4.1 \%$, respectively, as well as a $g_{\mathrm{EL}}$ of $-0.9 \times 10^{-3}$.

Owing to the excellent EL performances of AIDF molecules, it could be a promising approach to design efficient chiral polymers by combining AIDF units with side-chain type chiral groups. Two polymers P5 and P10 [81] (Fig. 15a) with different amounts of monomer units were synthesized by modified Suzuki coupling, with the calculated AIDF unit contents of $4.5 \%$ and $10.5 \%$ for P5 and P10, respectively. The PLQYs of P5 and P10 were $2.8 \%$ and $3.9 \%$ in THF, and $6.7 \%$ and $10.3 \%$ in solid states, respectively. P5 and P10 showed $k_{\text {RISC }}$ values of $2.07 \times 10^{6}$ and $2.50 \times 10^{6} \mathrm{~s}^{-1}$, and $\tau_{\text {delayed }}$ values of 1.358 and $1.366 \mu \mathrm{s}$, respectively, revealing favored TADF properties. In addition, the negative CPL signals for P5 and P10 of $-2.01 \times 10^{-3}$ and $-1.39 \times 10^{-3}$ were obtained (Fig. 15b). The introduction of 9-octyl-9H-carbazole moiety resulted in facile solubility and processability. The solution-processed OLED with a configuration of ITO/PEDOT: PSS $(50 \mathrm{~nm}) / 5 \mathrm{wt} \%$ P10: CBP $(50 \mathrm{~nm}) / \mathrm{TmPyPB}(45 \mathrm{~nm}) / \mathrm{LiF}(1 \mathrm{~nm}) / \mathrm{Al}$ emitted green $\mathrm{EL}$ with maximum $\mathrm{CE}, \mathrm{PE}$ and $\mathrm{EQE}$ of $2.52 \mathrm{~cd} \mathrm{~A}^{-2}, 0.94 \mathrm{~lm} \mathrm{~W}^{-1}$ and $0.87 \%$, respectively.

\section{AlEgens for luminescent solar concentrators}

Luminescent solar concentrators (LSCs), which were firstly conceived by Garwin in 1960 [82], rely on the absorption of incident solar light by luminescent materials embedded in glass or plastic substrates. Subsequently, the luminescent materials will emit photons and most of these photons will be trapped in the waveguide by total internal reflection. Then, photons are concentrated on the substrate edges by total internal

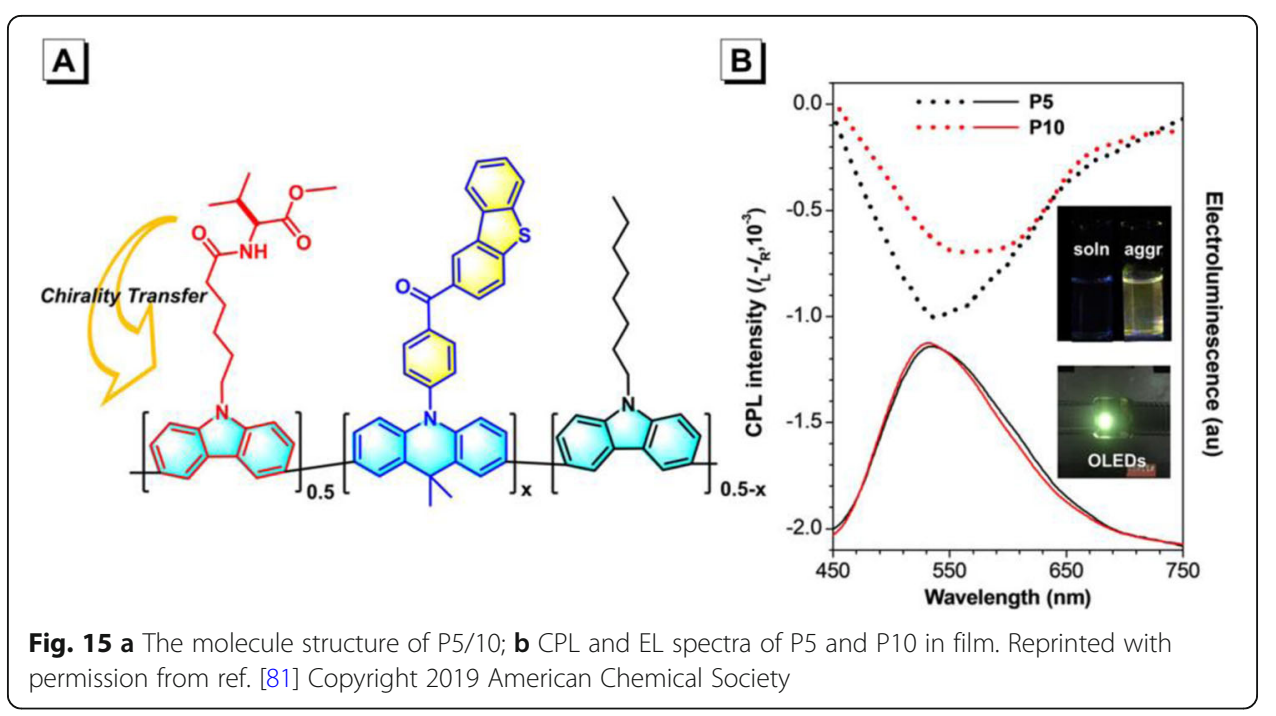


reflection [83]. Therefore, the solar cells can be placed at the edges to collect the concentrated light and produce electric power, which can reduce the area of photovoltaic cell and lower equipment cost. Currently, the performances of LSCs have been vastly limited by the reabsorption losses because the surrounding chromophores may reabsorb the emitted photons during the multiple internal reflection process [84]. Traditional chromophores used in LSCs, such as rhodamines and coumarins, generally have planar conjugated structures and suffer from both concentration quenching and self-absorption due to small Stokes shifts [85]. A common solution to this problem is to reduce the concentration of chromophores, but it means less light can be absorbed by the LSC at a given LSC device [86]. A more effective approach is to use AIEgen as the chromophore for LSCs. The twisted aromatic AIE materials usually have large Stokes shifts that can reduce reabsorption effectively. And no matter AIEgens are made into thin-films or dispersed in the matrix, it will not reduce the collection efficiency because they are free of concentration quenching. These distinctive advantages provide an important idea for the fabrication of highly efficient LSC devices.

Wong et al. first applied AIE-active materials for solar harvesting in LSC devices in $2014[87,88]$. They fabricated monolithic LSC devices by drop-casting $10 \mathrm{wt} \%$ of the star AIE-active material, TPE, in polymethyl methacrylate (PMMA). The PLQY of the drop-coating film was measured to be $40 \%$. The photophysical properties of the fluorophores in the LSC were closely related to the optical edge efficiency $\left(\eta_{\text {edge }}\right)$, which is defined as the ratio of the number of fluorescence photons that are waveguided to the edges relative to the number of the incident photons. The $\eta_{\text {edge }}$ of the LSC with TPE in PMMA, fabricated on glass substrates with dimensions of $10 \mathrm{~mm} \times 10 \mathrm{~mm} \times 1 \mathrm{~mm}$, was found to be $13.2 \%$. And the large Stokes shift of $1.11 \mathrm{eV}$ could effectively reduce reabsorption. Furthermore, other four TPE derivatives with extended conjugated skeletons were synthesized to generate better coupling with photovoltaic cells (Fig. 16). Subsequently, Wong et al. combined an excitation energy transfer (EET) strategy with

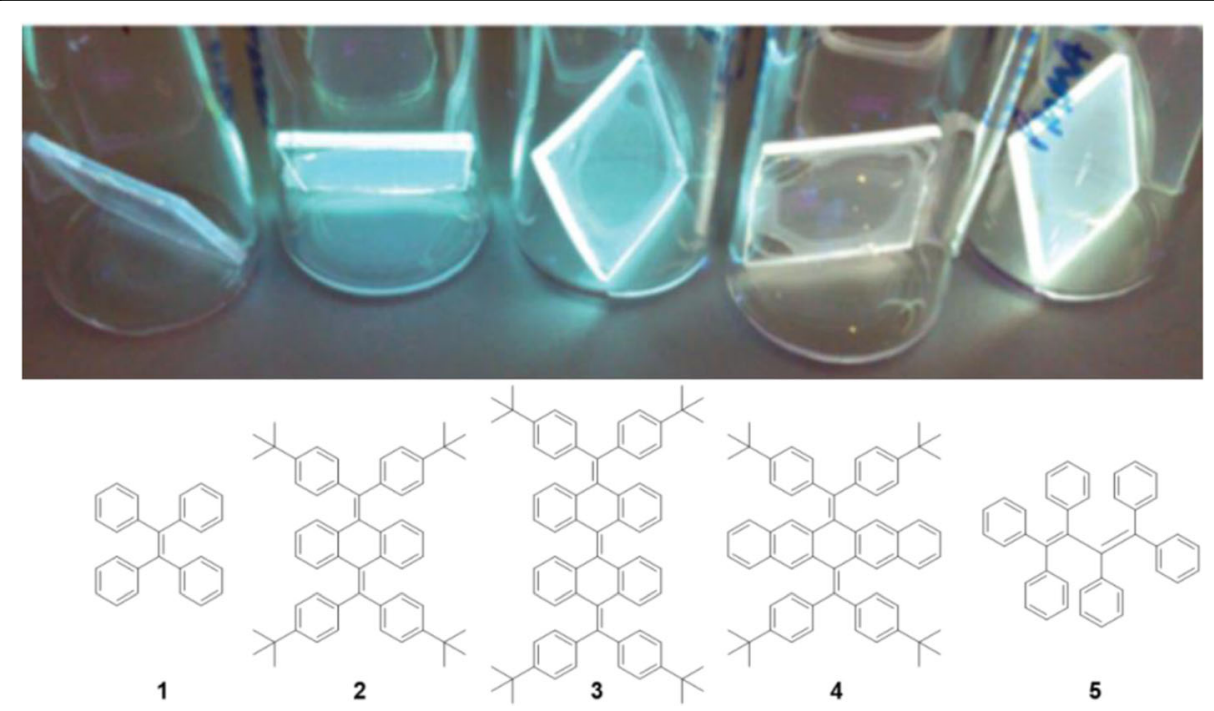

Fig. 16 Photos of PMMA films containing TPE and four TPE derivatives at 10\% w/w in PMMA upon excitation with a UV light source. Reprinted with permission from ref. [88] Copyright 2017 American Chemical Society 
the AIE-active donor to efficiently harvest light in the LSC. The branched donor DPATPAN displayed AIE behavior with a large Stokes shift and a high PLQY of 92\% (Fig. 17a) [89]. The small reabsorption loss of LSC was achieved through low absorption contribution of the acceptor. The experimental optical quantum efficiency (OQE) of LSC device based on DPATPAN-DCJTB system was about 58.2\%. However, the traditional acceptor DCJTB underwent concentration quenching, limiting their practical applications. Wong et al. [90] further replaced DCJTB with AIE-active groups, PITBTTPE, in LSCs (Fig. 17b). The emission spectrum of DPATPAN overlapped well with the absorption spectrum of PITBT-TPE, which was beneficial to energy migration. And the absorption spectrum of DPTPAN and the emission spectrum of PITBT-TPE was separated obviously which could avoid reabsorption of acceptor emission by the donor.

Pucci et al. [91] conducted an in-depth study on the utilization of red-emitting AIE materials in LSCs based on PMMA and polycarbonate (PC) (Fig. 18a). They dispersed a TPE derivative, TPE-AC, at $0.1-1.5 \mathrm{wt} \%$ contents in PC or PMMA. The films showed wide absorption bands at 400 and $550 \mathrm{~nm}$ and fluorescence emission peaking at 600$630 \mathrm{~nm}$. The LSC based on the red AIEgen exhibited a high optical efficiency $\left(\eta_{\text {opt }}\right)$ of 6.7\%. Afterwards, Pucci et al. [92] reported the AIEgen TPE_RED, an initiator to prepare red-emitting PMMA, i.e. PMMA_TPE_RED (Fig. 18b). In the work, TPE_RED initiated the polymerization of methyl methacrylate, and the polymer films of PMMA TPE_RED behaved an excellent PLQY of 26.5\%. The PMMA_TPE_RED film showed bright emission peaking at $650 \mathrm{~nm}$ with a large Stokes shift of about $170 \mathrm{~nm}$, which could control the reabsorption at a quite low level. The performance of LSC based on PMMA/PMMA_TPE_RED blend film showed the highest $\eta_{\text {opt }}$ of $10 \%$. The strategy of using well compatible and homogeneously distributed red-emitting AIEgens is a powerful method for gaining high-performance LSC devices.

\section{AIEgens for Electrofluorochromic devices}

In 1961, Platt reported the electrochromic (EC) phenomenon that electroactive materials can produce color changes between neutral state and colored switching state by electrochemical redox reactions [93]. So far, lots of EC materials have been reported and widely used in life, such as e-paper, smart windows, energy storage devices, etc.

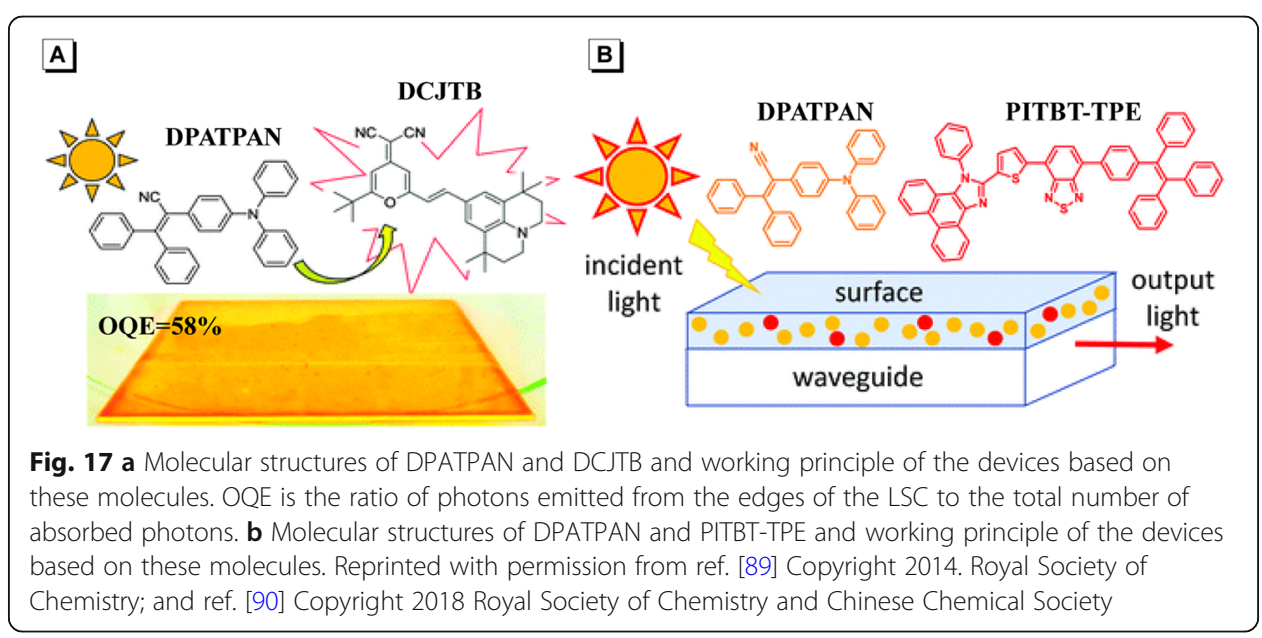


A

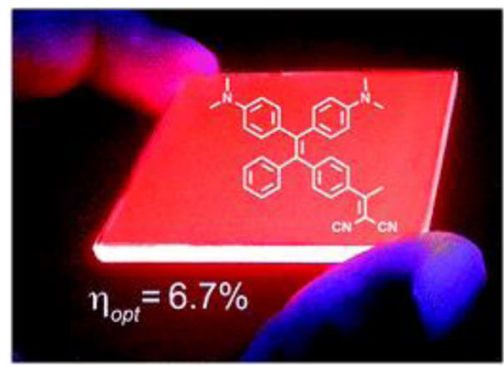

B

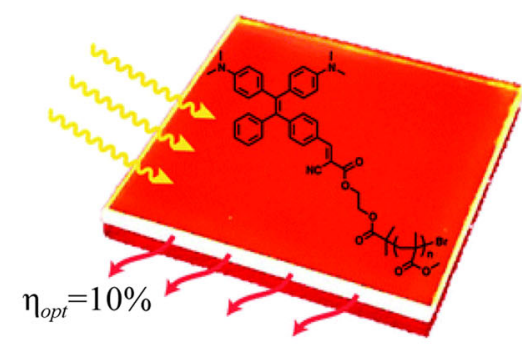

Fig. 18 a Chemical structure of red-emitting AIEgen TPE-AC and the performance of LSC based on TPE-AC. $\eta_{\text {opt }}$ is defined as the ratio between the short-circuit current measured in the case of the cell over the LSC edge and the short-circuit current of the bare cell when perpendicular to the light source); $\mathbf{b}$ Chemical structure of red-emitting AIEgen PMMA_TPE_RED and the performance of LSC based on PMMA_TPE_RED. Reprinted with permission from ref. [91] Copyright 2017 Royal Society of Chemistry and Chinese Chemical Society; and ref. [92] Copyright 2018 Royal Society of Chemistry

[94] Electrofluorochromism is a new concept combining electrochromism and fluorescence, which was first proposed by Lehn [95] in 1993. Subsequently, Audebert et al. [96] fabricated the first electrofluorochromic device based on tetrazine derivatives. However, it is regretful that the ACQ effect of conventional chromophores immensely restrict the emission contrast ratio which determines the performances of EFC devices. In contrast, AIEgens exhibit the unique advantage of avoiding concentration quenching and improving the emission contrast ratio to fabricate high-performance EFC devices.

Triphenylamine (TPA) derivatives are extensively applied in EFC devices due to their photoactive and electroactive properties [97-99]. Liou et al. reported several meaningful works by combining TPA units with AIEgens. Some AIE-active polymers were successfully prepared in 2018. The existence of TPA unit could effectively quench the emission of polyamides from neutral to oxidized states. And TPE unit was incorporated into the system for obtaining enhanced illumination capability. The EFC devices based on TPA-CN-CH/HV ( $n$-heptyl viologen) showed the highest contrast ratio $\left(I_{\text {off }} / I_{\text {on }}\right)$ of 105. The device based on TPA-OMe-TPE/HV exhibited the shortest response time of quenching process less than $4.9 \mathrm{~s}$. Whereas the response time of lighting processes was as long as $69 \mathrm{~s}$, which was not favorable for EFC devices [100]. Then, Liou et al. further synthesized two AIE-active materials, TPETPAOMe and BTOTPAOMe, to fabricate the EFC devices by a new technique of cross-linking gel-type. The EFC devices based on TPETPAOMe/HV behaved cyan-blue emission with a maximum peak at $505 \mathrm{~nm}$ under UV excitation, and the emission could be quenched by increasing the applied voltage from -0.10 to $1.60 \mathrm{~V}$ (Fig. 19). Upon applying potential back to $-0.10 \mathrm{~V}$, the devices showed bright emission again. The contrast ratios $\left(I_{\text {off }} / I_{\text {on }}\right)$ of the devices based on TPAOMe/HV and BTOTPAOMe/HV reached 6.89 and 6.66, respectively, and the response time of lighting processes was successfully shortened to $16.4 \mathrm{~s}$. Moreover, the electrochemical switching stability of the device based on TPE-TPAOMe/HV behaved well after 100 cycles [94]. Recently, Liou et al. further fabricated gel-type EFC devices based on diOMe-TPA-CN and diOMeTPA-CNBr and obtained a higher contrast ratio $\left(I_{\text {off }} / I_{\text {on }}\right)$ of $14.4[101]$.

Chen et al. [102] reported an AIE-active polyamide with diphenylamine (PA) and TPE units, achieving high contrast ratio and short response time simultaneously. As 
A<smiles>CCN(c1ccc(NC(=O)C2CCC(C(=O)C3CCCCC3)CC2)cc1)c1ccc(N(C)C)cc1</smiles>

TPA-CN-CH

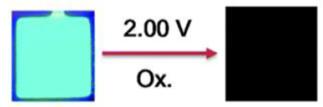

C<smiles>COc1ccc(N(c2ccc(OC)cc2)c2ccc(C(=C(c3ccccc3)c3ccccc3)c3ccccc3)cc2)cc1</smiles>

TPETPAOMe

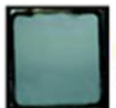

Oxidation

UV-irradiation
B<smiles>CCCCCCCN(c1ccc(NC(=O)c2ccc(C(=C(c3ccccc3)c3ccc(NC(=O)c4ccc(N(C)c5ccc(C)cc5)cc4)cc3)c3ccccc3)cc2)cc1)c1ccc(OC)cc1</smiles>

TPA-OMe-TPE

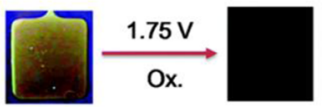

D
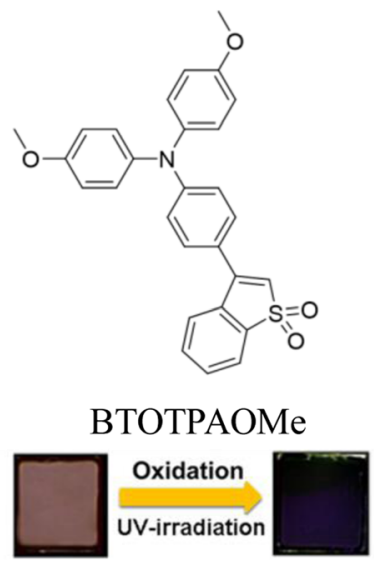

Fig. 19 a The molecular structure of TPA-CN-CH and the behaviors of EFC device based on TPA-CN-CH/HV with a specific applied voltage for oxidation. $\mathbf{b}$ The molecular structure of TPA-OMe-TPE and the behavior of EFC device based on TPA-OMe-TPE/HV with a specific applied voltage for oxidation. $\mathbf{c}$ The molecular structure of TPETPAOMe and the behavior of EFC device based on it with a specific applied voltage for oxidation. $\mathbf{d}$ The molecular structure of BTOTPAOMe and the behaviors of EFC device based on it with a specific applied voltage for oxidation. Reprinted with permission from ref. [100] Copyright 2018 Royal Society of Chemistry; and ref. [94] Copyright 2017 American Chemical Society

shown in Fig. 20, the PA-TPE film exhibited strong green-yellow emission in the neutral state, which was quickly quenched to dark when applying potential from 0 to $1 \mathrm{~V}$. This process showed the champion fluorescence on/off contrast ratio of 417 at that time, which was attributed to the high solid-state emission. The AIE-active polyamide was easy to generate porous film so that the devices showed faster response speed of fluorescence quenching and lighting processes. Subsequently, Chen et al. [103] synthesized polymer TPPA-TPE-PA to fabricate the EFC devices (Fig. 21), realizing the high fluorescence on/off contrast of up to 252, rapid response rate and excellent cycling stability over $10,000 \mathrm{~s}$.

\section{AlEgens for liquid crystal display}

Liquid crystal displays (LCDs) have got great progress over the past decades, displaying obvious advantages such as low power consumption, large display area, thin and light. Although LCDs have been widely used in our life, researchers are still committed to achieving performance of higher contrast, deeper color saturation, and lower power 


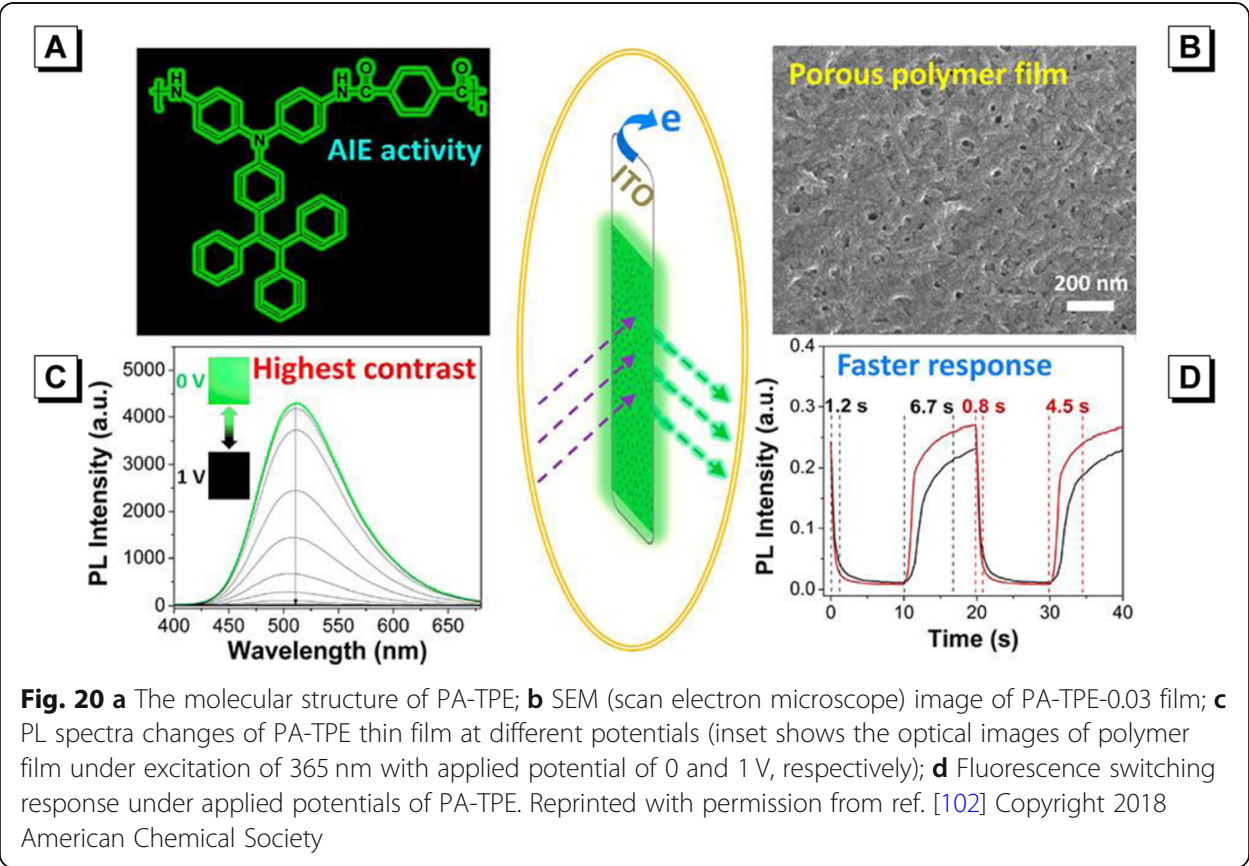

consumption. The light-emitting LCDs (LE-LCDs) based on luminescent liquid crystalline materials will be the promising alternatives to conventional LCDs [104, 105]. The commonly used approach to obtain LE-LCDs is to mix luminescent materials and nematic liquid crystals (LCs) with different concentrations. In order to achieve high contrast, high concentration of luminogens are usually required. However, conventional chromophores suffer from severe ACQ effect [106]. The unique advantages of AIEgens provide an exciting way to fabricate high-performance LE-LCDs.

Recently, it was found that TPE derivative, TPE-PPE, could emit anisotropically (Fig. 22a-c). The TPE-PPE/nematic LC mixture generated polarized luminescence and two types of LE-LCDs were fabricated. The first type LE-LCD device showed the image switching from nearly dark to bright yellow/green upon UV irradiation (Fig. 22d), which was easily controlled by electric field-off/on state. The strong color contrast and high solid-state emission were attributed to the native AIE property. As for the second type, different areas of the device could achieve

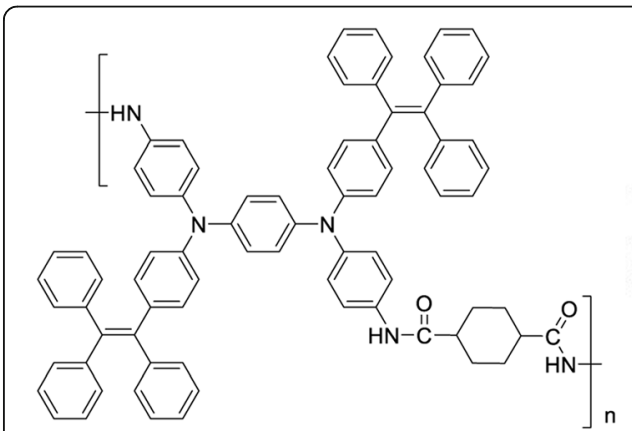

TPPA-TPE-PA

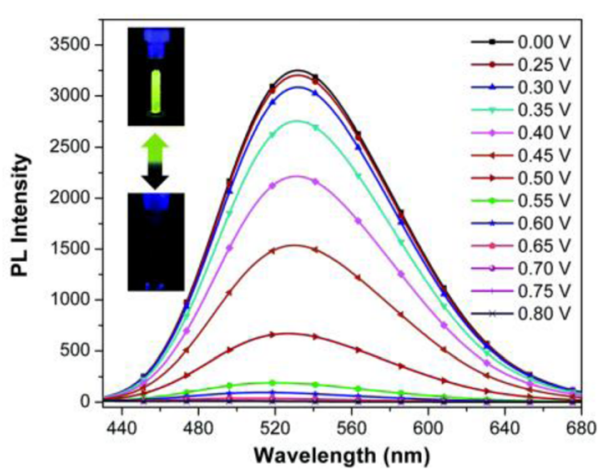

Fig. 21 The molecular structure of TPPA-TPE-PA and PL spectra changes of TPPA-TPE-PA thin film under different voltages. Reprinted with permission from ref. [103] Copyright 2019 Royal Society of Chemistry 

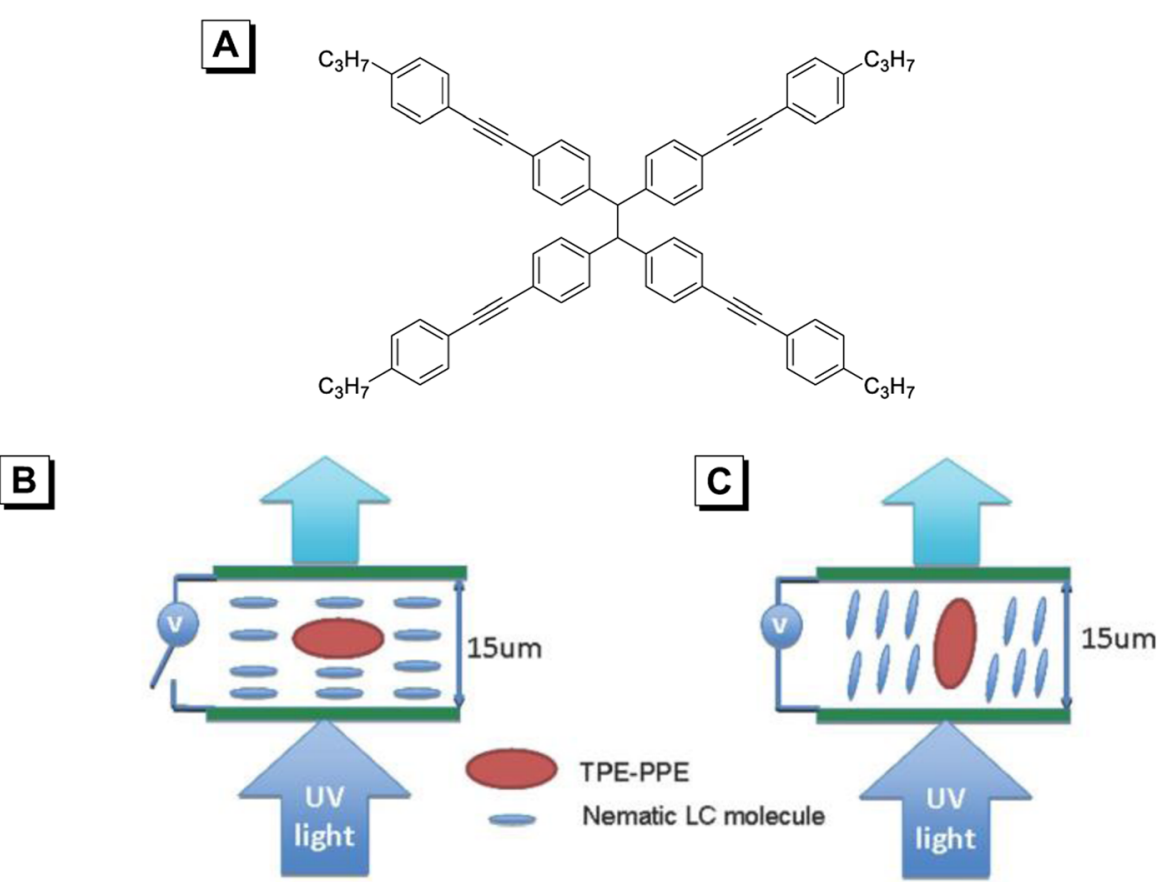

D

\section{Eoff}
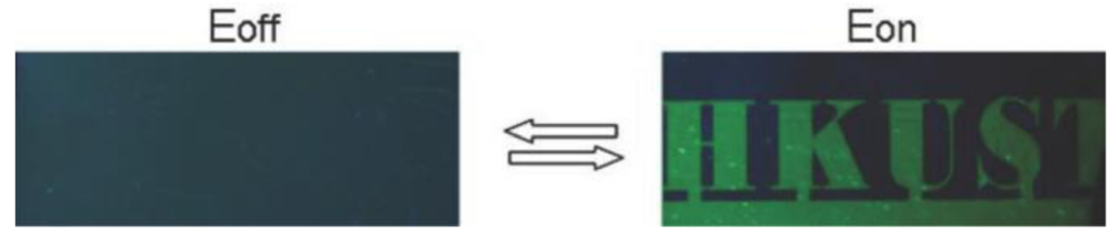

Fig. 22 a Chemical structure of TPE-PPE. b Electric field-off and c field-on (1 KHz, 8 v) states under UV irradiation. $\mathbf{d}$ Photograph of the LE-LCD device in the electric field-off and field-on states using lightemitting LC mixture. The LC mixture = Nematic LC PA $0182+0.1$ wt\% TPE-PPE. Reproduced with permission from ref. [107]. Copyright 2014 Wiley-VCH

transition between darkness and brightness in the electric field-off state by setting the polarizer in various directions. In the electric field-on state, all areas of the device would have same emission efficiency so that all images were disappeared (Fig. 23) [107]. TPE-PPE was introduced to chiral nematic LC, exhibiting AIE and CPL behaviors at the same time. The device showed yellow color due to Bragg reflection under sunlight and could emit green CPL light under UV light irradiation. The display device based on this system exhibited different region under different lighting conditions. With the electric field of $60 \mathrm{~V}$ applied on the device, the alignment of the LC molecules changed in the conductive ITO area. As a result, not only the Bragg reflection was broken, but also the CPL behavior disappeared and the PL intensity greatly dropped [108]. Recently, Cheng et al. [109] prepared Nematic Liquid Crystals ( $\mathrm{N}^{*}$-LC) by doping chiral AIE-active molecule, R/S-BINOL-CN, into achiral N-LC. BINOL-CN showed weak emission and almost no CPL signal in DMSO solution. But in the $\mathrm{KBr}$ pellet-dispersed solid state, bright yellow-green emission and high CPL signal at $536 \mathrm{~nm}$ were observed. The $\mathrm{N}^{*}$-LC displayed the maximum $g_{\text {lum }}$ values of about $-0.40 /+0.41$ at the concentration of $2.0 \mathrm{wt} \%$, achieving the CPL signal amplification (Fig. 24). 


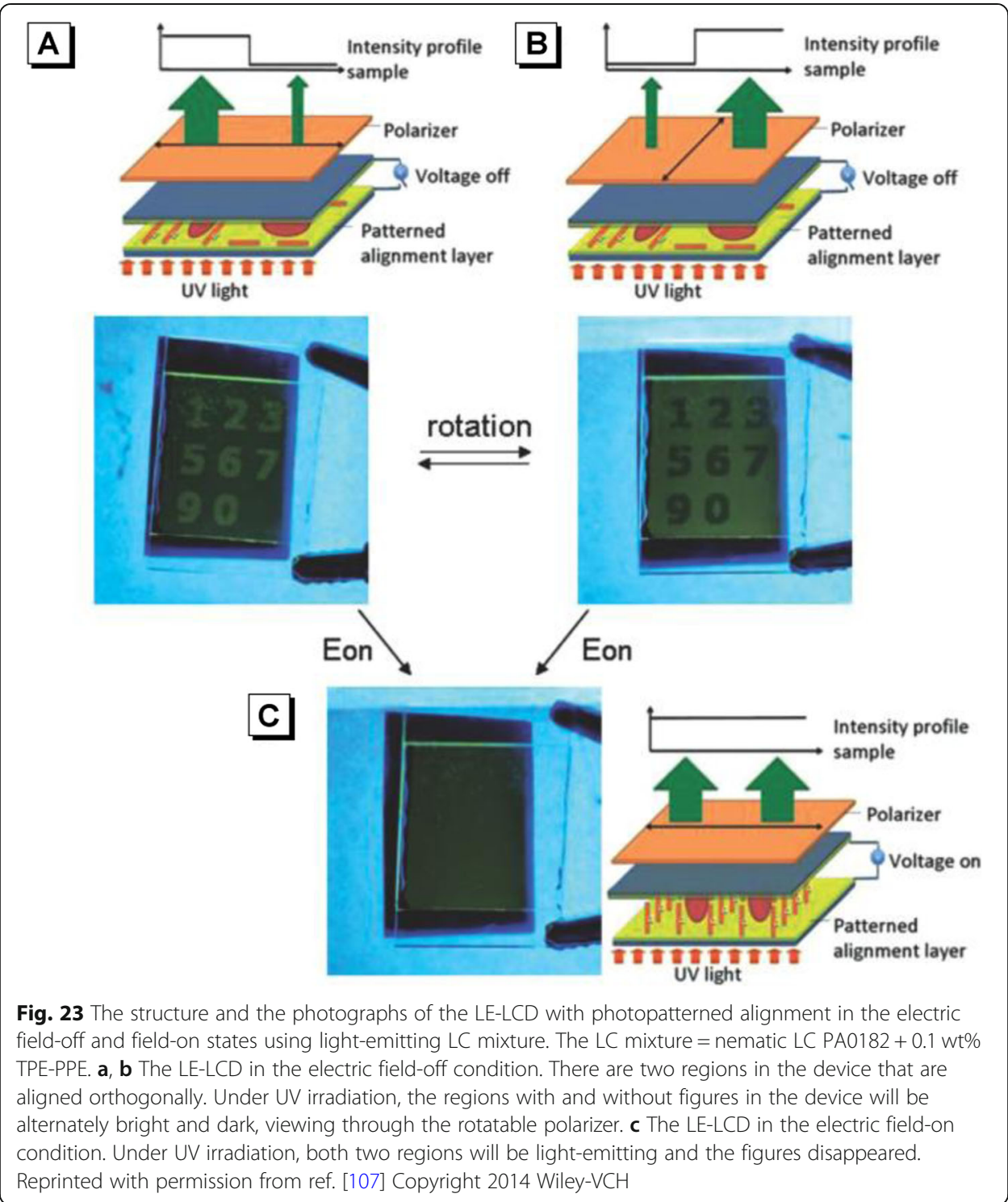

\section{Conclusion}

AIEgens have got rapid development and attracted great attentions in various fields over the last 20 years, owing to their unique photophysical properties in the aggregated state. Especially in the field of organic optoelectronics, functional materials derived from AIEgens have occupied the irreplaceable positions. In view of the significant achievements of AIEgens in recent years, this review presents representative advancements of AIEgens for the optoelectronic device applications of OLED, LSC, EFC and LCD. The structure-function relationship and the performances of optoelectronic devices are elucidated in detail. In OLEDs, based on the high PLQYs and flexible structure-tunability, blue AIEgens have been fully developed and applied in WOLEDs, host materials and full-color display. To exceed the exciton utilization of traditional fluorescent materials, delayed fluorescence property is integrated in AIEgens, achieving excellent EL efficiencies and negligible efficiency roll-off for nondoped OLEDs. With further decoration of CPL character on AIEgens, the OLEDs can emit circularly 


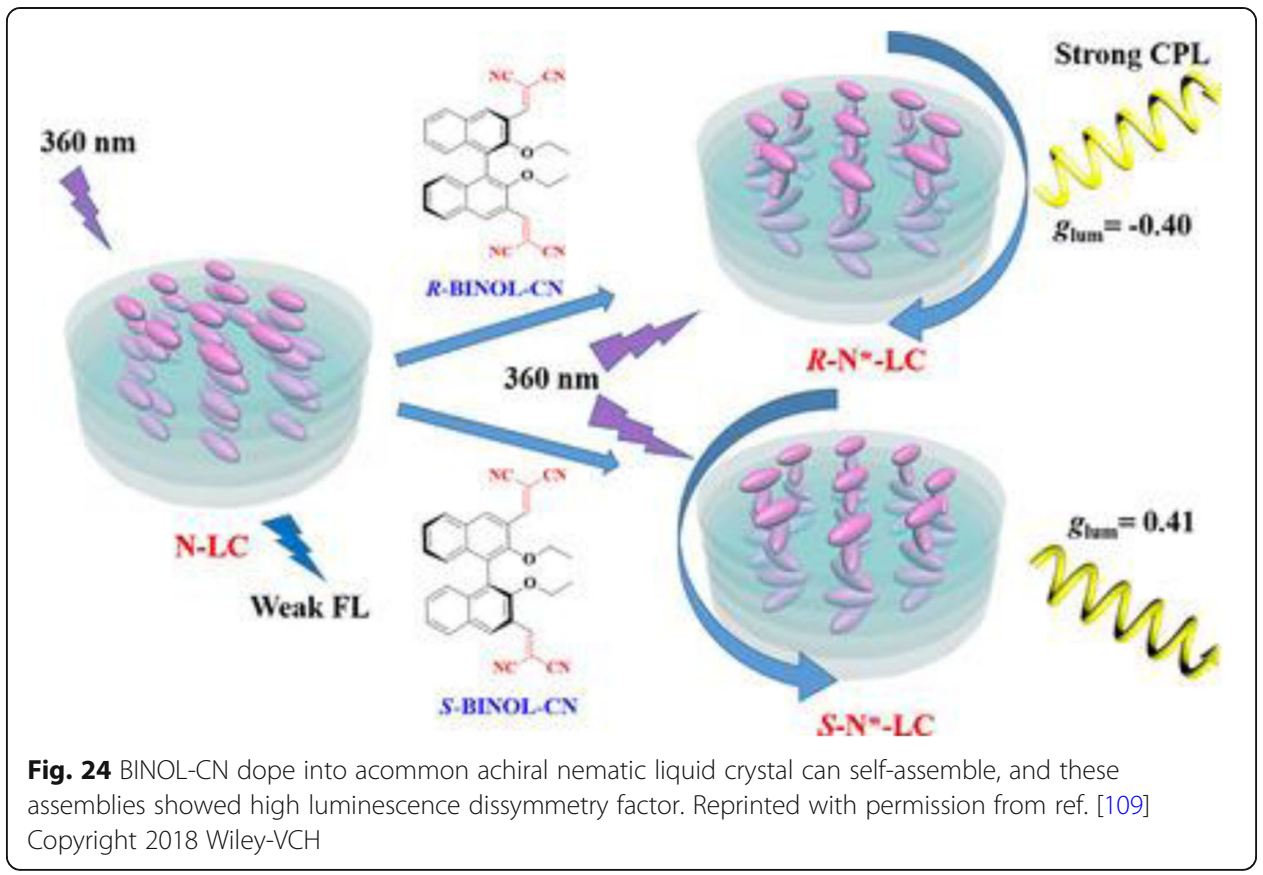

polarized light directly to avoid the energy loss caused by filters. To promote the commercialization of AIEgens in OLEDs, more efficient AIEgens with deep blue and red emissions are highly desired. The materials stability and device lifetime are important but still challenging. In LSCs, AIEgens with twisted aromatic structure can reduce the reabsorption effectively caused by the large Stokes shift as well as emission quenching by concentration or aggregation. At the same time, AIEgens can allow high concentrations in LSCs to improve the collection effect instead of suffering from the ACQ problem. In the following researches, adjusting the emission wavelength range to match different solar cells and improving cycling stability may become a research hotspot. In EFC devices, AIEgens are hopeful to improve the contrast ratio. The future work may focus on reducing the response time and increasing the stability of long-term operation of the device with a high contrast ratio. In LCDs, luminescent liquid crystals doped with AIEgens show exciting sparkle with self-luminous property. But there is still much room for further improvement in simplifying manufacturing process. Above all, we hope that this review can help researchers to explore more excellent AIEgen-based functional materials for the applications in organic optoelectronic fields as well as other research fronts.

\section{Acknowledgements}

This work was financially supported by the National Natural Science Foundation of China (21788102), the Natural Science Foundation of Guangdong Province (2019B030301003), and the Science and Technology Program of Guangzhou (201804020027).

\section{Author contributions}

Maoxing Yu, Ruishan Huang and Jingjing Guo contributed to manuscript writing. Zujin Zhao was responsible for funding acquisition, project design, administration and supervision, and manuscript writing and revision. Ben Zhong Tang was responsible for project administration and supervision. All authors have given approval to the final version of the manuscript.

Funding

National Natural Science Foundation of China (21788102).

Natural Science Foundation of Guangdong Province (2019B030301003).

Science and Technology Program of Guangzhou (201804020027). 
Availability of data and materials

All the data analyzed and discussed are cited from published articles. The figures are reproduced with permission.

\section{Competing interests}

The authors declare that thay have no competing interests.

\section{Author details}

${ }^{1}$ State Key Laboratory of Luminescent Materials and Devices, Guangdong Provincial Key Laboratory of Luminescence from Molecular Aggregates, South China University of Technology, Guangzhou 510640, China. ${ }^{2}$ Department of Chemistry, The Hong Kong University of Science \& Technology, Clear Water Bay, Kowloon, Hong Kong, China.

Received: 5 February 2020 Accepted: 17 March 2020

Published online: 03 April 2020

\section{References}

1. Clark J, Lanzani G. Organic photonics for communications. Nat Photonics. 2010:4:438-46.

2. Shirakawa H, Louis EJ, MacDiarmid AG, Chiang CK, Heeger AJ. Synthesis of electrically conducting organic polymers: halogen derivatives of polyacetylene, $(\mathrm{CH})_{x}$. J Chem Soc Chem Commun. 1977;16:578-80.

3. Tang CW, VanSlyke SA. Organic electroluminescent diodes. Appl Phys Lett. 1987;51:913-5.

4. Fahlman M, Fabiano S, Gueskine V, Simon D, Berggren M, Crispin X. Interfaces in organic electronics. Nat Rev Mater. 2019:4:627-50

5. Meredith GR, VanDusen J, Williams DJ. Optical and nonlinear optical characterization of molecularly doped thermotropic liquid crystalline polymers. Macromolecules. 1982;15:1385-9.

6. $\quad$ Tang CW. Two-layer organic photovoltaic cell. Appl Phys Lett. 1986;48:183-5.

7. Dong YQ, Lam JWY, Qin AJ, Liu JZ, Li Z, Tang BZ. Aggregation-induced emissions of tetraphenylethene derivatives and their utilities as chemical vapor sensors and in organic light-emitting diodes. Appl Phys Lett. 2007;91:011111.

8. Feringa BL, Jager WF, de Lange B. Organic materials for reversible optical data storage. Tetrahedron. 1993:49:8267-310.

9. Luo J, Xie Z, Lam JWY, Cheng L, Chen H, Qiu C, Kwok HS, Zhan X, Liu Y, Zhu D, Tang BZ. Aggregation-induced emission of 1-methyl-1,2,3,4,5-pentaphenylsilole. Chem Commun. 2001:1740-1.

10. Shen PC, Zhuang ZY, Zhao ZJ, Tang BZ. AlEgens based on main group heterocycles. J Mater Chem C. 2018;6:11835-52..

11. Mei J, Hong YN, Lam JWY, Qin AJ, Tang YH, Tang BZ. Aggregation-induced emission: the whole is more brilliant than the parts. Adv Mater. 2014;26:5429-79.

12. Mei J, Leung NLC, Kwok RTK, Lam JWY, Tang BZ. Aggregation-induced emission: together we Shine, united we soar! Chem Rev. 2015;115:11718-940.

13. Zhao ZJ, He BR, Tang B. Aggregation-induced emission of siloles. Chem Sci. 2015;6:5347-65.

14. Guo J, Zhao Z, Tang BZ. Purely organic materials with aggregation-induced delayed fluorescence for efficient nondoped OLEDs. Adv Opt Mater. 2018;6:1800264.

15. Zhang XY, Wang K, Liu MY, Zhang XQ, Tao L, Chen YW, Wei Y. Polymeric AlE-based nanoprobes for biomedical applications: recent advances and perspectives. Nanoscale. 2015;7:11486-508.

16. Ding D, Li K, Liu B, Tang BZ. Bioprobes based on AlE Fluorogens. Acc Chem Res. 2013;46:2441-53.

17. Li J, Shen P, Zhao Z, Tang BZ. Through-space conjugation: a thriving alternative for optoelectronic materials. CCS Chem. 2019;1:181-96.

18. Sun Y, Forrest SR. Enhanced light out-coupling of organic light-emitting devices using embedded low-index grids. Nat Photonics. 2008;2:483-7.

19. Koo WH, Jeong SM, Araoka F, Ishikawa K, Nishimura S, Toyooka T, Takezoe H. Light extraction from organic lightemitting diodes enhanced by spontaneously formed buckles. Nat Photonics. 2010;4:222-6.

20. Reineke S, Lindner F, Schwartz G, Seidler N, Walzer K, Lussem B, Leo K. White organic light-emitting diodes with fluorescent tube efficiency. Nature. 2009;459:234-U116.

21. Baldo MA, O'Brien DF, Thompson ME, Forrest SR. Excitonic singlet-triplet ratio in a semiconducting organic thin film. Phys Rev B. 1999;60:14422-8.

22. Li W, Pan Y, Xiao R, Peng Q, Zhang S, Ma D, Li F, Shen F, Wang Y, Yang B, Ma Y. Employing 100\% Excitons in OLEDs by utilizing a fluorescent molecule with hybridized local and charge-transfer excited state. Adv Funct Mater. 2014;24:1609-14.

23. Yang D, Duan P, Liu M. Dual Upconverted and Downconverted circularly polarized luminescence in donor-acceptor assemblies. Angew Chem Int Ed. 2018;57:9357-61.

24. Chan CY, Tanaka M, Nakanotani H, Adachi C. Efficient and stable sky-blue delayed fluorescence organic light-emitting diodes with ClEy below 0.4. Nat Commun. 2018;9:5036.

25. Farinola GM, Ragni R. Electroluminescent materials for white organic light emitting diodes. Chem Soc Rev. 2011;40: 3467-82.

26. Zhu M, Yang C. Blue fluorescent emitters: design tactics and applications in organic light-emitting diodes. Chem Soc Rev. 2013:42:4963-76.

27. Lee J, Chen C, Lee P, Lin H, Leung M, Chiu T, Lin C. Blue organic light-emitting diodes: current status, challenges, and future outlook. J Mater Chem C. 2019;7:5874-88.

28. Cai X, Su S. Marching toward highly efficient, pure-blue, and stable thermally activated delayed fluorescent organic light-emitting diodes. Adv Funct Mater. 2018;28:1802558.

29. Shi JM, Tang CW. Anthracene derivatives for stable blue-emitting organic electroluminescence devices. Appl Phys Lett. 2002;80:3201-3.

30. Chen B, Liu B, Zeng J, Nie H, Xiong Y, Zou J, Ning H, Wang Z, Zhao Z, Tang BZ. Efficient bipolar blue AlEgens for highperformance nondoped blue OLEDs and hybrid White OLEDs. Adv Funct Mater. 2018;28:1803369. 
31. Li Y, Xu Z, Zhu X, Chen B, Wang Z, Xiao B, Lam JWY, Zhao Z, Ma D, Tang BZ. Creation of efficient blue aggregationinduced emission Luminogens for high-performance nondoped blue OLEDs and hybrid White OLEDs. ACS Appl Mater Interfaces. 2019;11:17592-601.

32. Zhan X, Wu Z, Lin Y, Xie Y, Peng Q, Li Q, Ma D, Li Z. Benzene-cored AlEgens for deep-blue OLEDs: high performance without hole-transporting layers, and unexpected excellent host for orange emission as a side-effect. Chem Sci. 2016;7:4355-63.

33. Xu Z, Gong Y, Dai Y, Sun Q, Qiao X, Yang D, Zhan X, Li Z, Tang BZ, Ma D. High efficiency and low roll-off hybrid WOLEDs by using a deep blue aggregation-induced emission material simultaneously as blue emitter and phosphor host. Adv Opt Mater. 2019;7:1801539.

34. Xu Z, Gu J, Qiao X, Qin A, Tang BZ, Ma D. Highly efficient deep blue aggregation-induced emission organic molecule: a promising multifunctional electroluminescence material for blue/green/Orange/red/White OLEDs with superior efficiency and low roll-off. ACS Photonics. 2019;6:767-78.

35. Liu B, Yu Z, He D, Zhu Z, Zheng J, Yu Y, Xie W, Tong Q, Lee C. Ambipolar D-A type bifunctional materials with hybridized local and charge-transfer excited state for high performance electroluminescence with EQE of $7.20 \%$ and CIEy 0.06. J Mater Chem C. 2017;5:5402-10.

36. Li W, Liu D, Shen F, Ma D, Wang Z, Feng T, Xu Y, Yang B, Ma Y. A twisting donor-acceptor molecule with an intercrossed excited state for highly efficient, Deep-Blue Electroluminescence. Adv Funct Mater. 2012;22:2797-803.

37. Zhang H, Zeng J, Luo W, Wu H, Zeng C, Zhang K, Feng W, Wang Z, Zhao Z, Tang BZ. Synergistic tuning of the optical and electrical performance of AlEgens with a hybridized local and charge-transfer excited state. J Mater Chem C. 2019;7:6359-68.

38. Chiang CJ, Kimyonok A, Etherington MK, Griffiths GC, Jankus V, Turksoy F, Monkman AP. Ultrahigh efficiency fluorescent single and bi-layer organic light emitting diodes: the key role of triplet fusion. Adv Funct Mater. 2013;23:739-46.

39. Tang, X., Bai, Q., Shan, T., Li, J., Gao, Y., Liu, F., Liu, H., Peng, Q., Yang, B., Li, F., Lu, P.: Efficient Nondoped Blue Fluorescent Organic Light-Emitting Diodes (OLEDs) with a High External Quantum Efficiency of 9.4\% @ $1000 \mathrm{~cd} \mathrm{~m}^{-2}$ Based on Phenanthroimidazole-Anthracene Derivative. Adv Funct Mater 28, 1705813 (2018)

40. Bian M, Zhao Z, Li Y, Li Q, Chen Z, Zhang D, Wang S, Bian Z, Liu Z, Duan L, Xiao L. A combinational molecular design to achieve highly efficient deep-blue electrofluorescence. J Mater Chem C. 2018;6:745-53.

41. Chaskar A, Chen HF, Wong KT. Bipolar host materials: a chemical approach for highly efficient electrophosphorescent devices. Adv Mater. 2011;23:3876-95.

42. Kim D, Coropceanu V, Bredas JL. Design of efficient ambipolar host materials for organic blue electrophosphorescence: theoretical characterization of hosts based on carbazole derivatives. J Am Chem Soc. 2011;133:17895-900.

43. Lee CW, Lee JY. High quantum efficiency in solution and vacuum processed blue phosphorescent organic light emitting diodes using a novel benzofuropyridine-based bipolar host material. Adv Mater. 2013;25:596-600.

44. Hu S, Zeng J, Zhu X, Guo J, Chen S, Zhao Z, Tang BZ. Universal bipolar host materials for blue, green, and red phosphorescent OLEDs with excellent efficiencies and small-efficiency roll-off. ACS Appl Mater Interfaces. 2019;11:27134-44

45. Nakanotani H, Higuchi T, Furukawa T, Masui K, Morimoto K, Numata M, Tanaka H, Sagara Y, Yasuda T, Adachi C. Highefficiency organic light-emitting diodes with fluorescent emitters. Nat Commun. 2014;5:4016.

46. Xie Y, Gong Y, Han M, Zhang F, Peng Q, Xie G, Li Z. Tetraphenylcyclopentadiene-based Hyperbranched polymers: convenient syntheses from one pot "A4 + B2" polymerization and high external quantum yields up to $9.74 \%$ in OLED devices. Macromolecules. 2019;52:896-903.

47. Kim D, D'Aléo A, Chen X, Sandanayaka ADS, Yao D, Zhao L, Komino T, Zaborova E, Canard G, Tsuchiya Y, Choi E, Wu JW Fages F, Brédas J, Ribierre J, Adachi C. High-efficiency electroluminescence and amplified spontaneous emission from a thermally activated delayed fluorescent near-infrared emitter. Nat Photonics. 2018;12:98-104.

48. Uoyama H, Goushi K, Shizu K, Nomura H, Adachi C. Highly efficient organic light-emitting diodes from delayed fluorescence. Nature. 2012;492:234-8.

49. Goushi K, Yoshida K, Sato K, Adachi C. Organic light-emitting diodes employing efficient reverse intersystem crossing for triplet-to-singlet state conversion. Nat Photonics. 2012;6:253-8.

50. Yang Z, Mao Z, Xie Z, Zhang Y, Liu S, Zhao J, Xu J, Chi Z, Aldred MP. Recent advances in organic thermally activated delayed fluorescence materials. Chem Soc Rev. 2017:46:915-1016.

51. Godumala M, Choi S, Cho MJ, Choi DH. Recent breakthroughs in thermally activated delayed fluorescence organic light emitting diodes containing non-doped emitting layers. J Mater Chem C. 2019;7:2172-98

52. Jeon SK, Lee HL, Yook KS, Lee JY. Recent Progress of the lifetime of organic light-emitting diodes based on thermally activated delayed fluorescent material. Adv Mater. 2019;31:1803524.

53. Furue R, Nishimoto T, Park IS, Lee J, Yasuda T. Aggregation-induced delayed fluorescence based on donor/acceptor-tethered Janus Carborane triads: unique Photophysical properties of nondoped OLEDs. Angew Chem Int Ed. 2016;55:7171-5.

54. Aizawa N, Tsou CJ, Park IS, Yasuda T. Aggregation-induced delayed fluorescence from phenothiazine-containing donoracceptor molecules for high-efficiency non-doped organic light-emitting diodes. Polym J. 2016;49:197-202..

55. Guo J, Li X, Nie H, Luo W, Gan S, Hu S, Hu R, Qin A, Zhao Z, Su S, Tang BZ. Achieving high-performance nondoped OLEDs with extremely small efficiency roll-off by combining aggregation-induced emission and thermally activated delayed fluorescence. Adv Funct Mater. 2017;27:1606458.

56. Guo J, Li X, Nie H, Luo W, Hu R, Qin A, Zhao Z, Su S, Tang BZ. Robust luminescent materials with prominent aggregation-induced emission and thermally activated delayed fluorescence for high-performance organic lightemitting diodes. Chem Mater. 2017;29:3623-31.

57. Huang J, Nie H, Zeng J, Zhuang Z, Gan S, Cai Y, Guo J, Su SJ, Zhao Z, Tang BZ. Highly efficient nondoped OLEDs with negligible efficiency roll-off fabricated from aggregation-induced delayed fluorescence Luminogens. Angew Chem Int Ed. 2017;56:12971-6.

58. Liu H, Zeng J, Guo J, Nie H, Zhao Z, Tang BZ. High-performance non-doped OLEDs with nearly $100 \%$ Exciton use and negligible efficiency roll-off. Angew Chem Int Ed. 2018:57:9290-4.

59. Guo J, Fan J, Lin L, Zeng J, Liu H, Wang CK, Zhao Z, Tang BZ. Mechanical insights into aggregation-induced delayed fluorescence materials with anti-kasha behavior. Adv Sci. 2019;6:1801629.

60. Gan S, Hu S, Li XL, Zeng J, Zhang D, Huang T, Luo W, Zhao Z, Duan L, Su SJ, Tang BZ. Heavy atom effect of bromine significantly enhances Exciton utilization of delayed fluorescence Luminogens. ACS Appl Mater Interfaces. 2018;10: 17327-34. 
61. Xiang Y, Zhao Y, Xu N, Gong S, Ni F, Wu K, Luo J, Xie G, Lu Z, Yang C. Halogen-induced internal heavy-atom effect shortening the emissive lifetime and improving the fluorescence efficiency of thermally activated delayed fluorescence emitters. J Mater Chem C. 2017;5:12204-10.

62. Xu J, Zhu X, Guo J, Fan J, Zeng J, Chen S, Zhao Z, Tang BZ. Aggregation-Induced Delayed Fluorescence Luminogens with Accelerated Reverse Intersystem Crossing for High-Performance OLEDs. ACS Materials Lett. 2019;1:613-9.

63. Zhao H, Wang Z, Cai X, Liu K, He Z, Liu X, Cao Y, Su S. Highly efficient thermally activated delayed fluorescence materials with reduced efficiency roll-off and low on-set voltages. Mater Chem Front. 2017;1:2039-46.

64. Thangaraji V, Rajamalli P, Jayakumar J, Huang MJ, Chen YW, Cheng CH. Quinolinylmethanone-based thermally activated delayed fluorescence emitters and the application in OLEDs: effect of Intramolecular H-bonding. ACS Appl Mater Interfaces. 2019;11:17128-33.

65. Zhang Q, Sun S, Liu W, Leng P, Lv X, Wang Y, Chen H, Ye S, Zhuang S, Wang L. Integrating TADF luminogens with AlE characteristics using a novel acridine-carbazole hybrid as donor for high-performance and low efficiency roll-off OLEDs. J Mater Chem C. 2019;7:9487-95.

66. Xiang S, Huang Z, Sun S, Lv X, Fan L, Ye S, Chen H, Guo R, Wang L. Highly efficient non-doped OLEDs using aggregation-induced delayed fluorescence materials based on 10-phenyl-10H-phenothiazine 5,5-dioxide derivatives. J Mater Chem C. 2018;6:11436-43.

67. Yang Z, Mao Z, Xu C, Chen X, Zhao J, Yang Z, Zhang Y, Wu W, Jiao S, Liu Y, Aldred MP, Chi Z. A sterically hindered asymmetric D-A-D' thermally activated delayed fluorescence emitter for highly efficient non-doped organic lightemitting diodes. Chem Sci. 2019;10:8129-34.

68. Zhang P, Zeng J, Guo J, Zhen S, Xiao B, Wang Z, Zhao Z, Tang BZ. New aggregation-induced delayed fluorescence Luminogens with through-space charge transfer for efficient non-doped OLEDs. Front Chem. 2019;7:199.

69. Wu K, Wang Z, Zhan L, Zhong C, Gong S, Xie G, Yang C. Realizing highly efficient solution-processed Homojunction-like sky-blue OLEDs by using thermally activated delayed fluorescent emitters featuring an aggregation-induced emission property. J Phys Chem Lett. 2018;9:1547-53.

70. Huang J, Xu Z, Cai Z, Guo J, Guo J, Shen P, Wang Z, Zhao Z, Ma D, Tang BZ. Robust luminescent small molecules with aggregation-induced delayed fluorescence for efficient solution-processed OLEDs. J Mater Chem C. 2019;7:330-9.

71. Roose J, Tang BZ, Wong KS. Circularly-polarized luminescence (CPL) from chiral AIE molecules and macrostructures. Small. 2016;12:6495-512.

72. Brandt JR, Salerno F, Fuchter MJ. The added value of small-molecule chirality in technological applications. Nat Rev Chem. 2017;1:0045

73. Feng H, Liu C, Li Q, Zhang H, Lam JWY, Tang BZ. Structure, assembly, and function of (latent)-chiral AlEgens. ACS Materials Lett. 2019;1:192-202.

74. Han J, Guo S, Lu H, Liu S, Zhao Q, Huang W. Recent Progress on circularly polarized luminescent materials for organic optoelectronic devices. Adv Opt Mater. 2018:6:1800538.

75. Song F, Xu Z, Zhang Q, Zhao Z, Zhang H, Zhao W, Qiu Z, Qi C, Zhang H, Sung HHY, Williams ID, Lam JWY, Zhao Z, Qin A, Ma D, Tang BZ. Highly efficient circularly polarized electroluminescence from aggregationinduced emission Luminogens with amplified chirality and delayed fluorescence. Adv Funct Mater. 2018;28: 1800051.

76. Wu Z, Han H, Yan Z, Luo X, Wang Y, Zheng Y, Zuo J, Pan Y. Chiral Octahydro-Binaphthol Compound-Based Thermally Activated Delayed Fluorescence Materials for Circularly Polarized Electroluminescence with Superior EQE of 32.6\% and Extremely Low Efficiency Roll-Off. Adv Mater. 2019;31:1900524.

77. Wu Z, Yan Z, Luo X, Yuan L, Liang W, Wang Y, Zheng Y, Zuo J, Pan Y. Non-doped and doped circularly polarized organic light-emitting diodes with high performances based on chiral octahydro-binaphthyl delayed fluorescent luminophores. J Mater Chem C. 2019;7:7045-52.

78. Li M, Li SH, Zhang D, Cai M, Duan L, Fung MK, Chen CF. Stable enantiomers displaying thermally activated delayed fluorescence: efficient OLEDs with circularly polarized electroluminescence. Angew Chem Int Ed. 2018;57:2889-93.

79. Yang L, Zhang Y, Zhang X, Li N, Quan Y, Cheng Y. Doping-free circularly polarized electroluminescence of AlE-active chiral binaphthyl-based polymers. Chem Commun. 2018;54:9663-6.

80. Wang Y, Zhang Y, Hu W, Quan Y, Li Y, Cheng Y. Circularly polarized electroluminescence of thermally activated delayed fluorescence-active chiral Binaphthyl-based Luminogens. ACS Appl Mater Interfaces. 2019;11:26165-73.

81. Hu Y, Song F, Xu Z, Tu Y, Zhang H, Cheng Q, Lam JWY, Ma D, Tang BZ. Circularly polarized luminescence from chiral conjugated poly (carbazole-ran-acridine) s with aggregation-induced emission and delayed fluorescence. ACS Appl Polym Mater. 2019;1:221-9.

82. Garwin RL. The collection of light from scintillation counters. Rev Sci Instrum. 1960;31:1010-1.

83. Wilson LR, Rowan BC, Robertson N, Moudam O, Jones AC, Richards BS. Characterization and reduction of reabsorption losses in luminescent solar concentrators. Appl Opt. 2010;49:1651-61.

84. Goetzberger A, Greube W. Solar energy conversion with fluorescent collectors. Appl Phys. 1977;14:123-39.

85. Haines C, Chen M, Ghiggino KP. The effect of perylene diimide aggregation on the light collection efficiency of luminescent concentrators. Sol Energy Mater Sol Cells. 2012;105:287-92.

86. Debije MG, Verbunt PPC. Thirty years of luminescent solar concentrator research: solar energy for the built environment. Adv Energy Mater. 2012;2:12-35.

87. Banal JL, White JM, Ghiggino KP, Wong WWH. Concentrating aggregation-induced fluorescence in planar waveguides: a proof-of-principle. Sci Rep. 2014;4:4635.

88. Banal JL, Zhang B, Jones DJ, Ghiggino KP, Wong WWH. Emissive molecular aggregates and energy migration in luminescent solar concentrators. Acc Chem Res. 2017:50:49-57.

89. Banal JL, Ghiggino KP, Wong WWH. Efficient light harvesting of a luminescent solar concentrator using excitation energy transfer from an aggregation-induced emitter. Phys Chem Chem Phys. 2014;16:25358-63.

90. Zhang B, Banal JL, Jones DJ, Tang BZ, Ghiggino KP, Wong WWH. Aggregation-induced emission-mediated spectral downconversion in luminescent solar concentrators. Mater Chem Front. 2018;2:615-9.

91. De Nisi F, Francischello R, Battisti A, Panniello A, Fanizza E, Striccoli M, Gu X, Leung NLC, Tang BZ, Pucci A. Red-emitting AlEgen for luminescent solar concentrators. Mater Chem Front. 2017;1:1406-12. 
92. Mori R, lasilli G, Lessi M, Muñoz-García AB, Pavone M, Bellina F, Pucci A. Luminescent solar concentrators based on PMMA films obtained from a red-emitting ATRP initiator. Polym Chem. 2018;9:1168-77.

93. Platt JR. Electrochromism, a possible change of color producible in dyes by an electric field. J Chem Phys. 1961;34:862-3.

94. Lin HT, Huang CL, Liou GS. Design, synthesis, and Electrofluorochromism of new Triphenylamine derivatives with AIEactive pendent groups. ACS Appl Mater Interfaces. 2019;11:11684-90.

95. Goulle V, Harriman A, Lehn JM. An electro-photoswitch: redox switching of the luminescence of a bipyridine metal complex. J Chem Soc, Chem Commun. 1993:1034-6.

96. Kim Y, Kim E, Clavier G, Audebert P. New tetrazine-based fluoroelectrochromic window; modulation of the fluorescence through applied potential. Chem Commun. 2006:3612-4.

97. Ning Z, Chen Z, Zhang Q, Yan Y, Qian S, Cao Y, Tian H. Aggregation-induced emission (AlE)-active starburst Triarylamine Fluorophores as potential non-doped red emitters for organic light-emitting diodes and $\mathrm{Cl}_{2}$ gas Chemodosimeter. $\mathrm{Adv}$ Funct Mater. 2007;17:3799-807.

98. Hsiao S, Cheng S. New electroactive and electrochromic aromatic polyamides with ether-linked bis (triphenylamine) units. J Polym Sci Part A: Polym Chem. 2015;53:496-510.

99. Wu J, Liou GS. High-performance Electrofluorochromic devices based on Electrochromism and photoluminescenceactive novel poly (4-Cyanotriphenylamine). Adv Funct Mater. 2014;24:6422-9.

100. Cheng S, Han T, Huang TY, Tang BZ, Liou GS. High-performance electrofluorochromic devices based on aromatic polyamides with AlE-active tetraphenylethene and electro-active triphenylamine moieties. Polym Chem. 2018;9:4364-73.

101. Chen SY, Chiu YW, Liou GS. Substituent effects of AIE-active alpha-cyanostilbene-containing triphenylamine derivatives on electrofluorochromic behavior. Nanoscale. 2019;11:8597-603.

102. Sun N, Su K, Zhou Z, Yu Y, Tian X, Wang D, Zhao X, Zhou H, Chen C. AlE-active polyamide containing diphenylamineTPE moiety with superior Electrofluorochromic performance. ACS Appl Mater Interfaces. 2018;10:16105-12.

103. Sun N, Su K, Zhou Z, Tian X, Wang D, Vilbrandt N, Fery A, Lissel F, Zhao X, Chen C. Synergistic effect between electroactive tetraphenyl-p-phenylenediamine and AIE-active tetraphenylethylene for highly integrated electrochromic/ electrofluorochromic performances. J Mater Chem C. 2019;7:9308-15.

104. Wang L, Dong H, Li Y, Liu R, Wang YF, Bisoyi HK, Sun LD, Yan CH, Li Q. Luminescence-driven reversible handedness inversion of self-organized helical superstructures enabled by a novel near-infrared light nanotransducer. Adv Mater. 2015;27:2065-9.

105. Li H, Cheng J, Zhao Y, Lam JWY, Wong KS, Wu H, Li B, Tang BZ. L-Valine methyl ester-containing tetraphenylethene: aggregation-induced emission, aggregation-induced circular dichroism, circularly polarized luminescence, and helical self-assembly. Mater Horiz. 2014;1:518-21.

106. Mishra A, Ma C, Bäuerle P. Functional Oligothiophenes: molecular Design for Multidimensional Nanoarchitectures and Their Applications. Chem Rev. 2009;109:1141-276.

107. Zhao D, Fan F, Cheng J, Zhang Y, Wong KS, Chigrinov VG, Kwok HS, Guo L, Tang BZ. Light-emitting liquid crystal displays based on an aggregation-induced emission Luminogen. Adv Opt Mater. 2015;3:199-202.

108. Zhao D, Fan F, Chigrinov VG, Kwok HS, Tang BZ. Aggregate-induced emission in light-emitting liquid crystal display technology. J Soc Inf Display. 2015;23:218-22.

109. Li X, Li Q, Wang Y, Quan Y, Chen D, Cheng Y. Strong aggregation-induced CPL response promoted by chiral emissive Nematic liquid crystals (N*-LCs). Chem Eur J. 2018;24:12607-12.

\section{Publisher's Note}

Springer Nature remains neutral with regard to jurisdictional claims in published maps and institutional affiliations.

\section{Submit your manuscript to a SpringerOpen ${ }^{\circ}$ journal and benefit from:}

- Convenient online submission

- Rigorous peer review

- Open access: articles freely available online

- High visibility within the field

- Retaining the copyright to your article

Submit your next manuscript at $\boldsymbol{\nabla}$ springeropen.com 